\title{
Abstracts of the 25th Annual Meeting of ESPACOMP, the International Society for Medication Adherence, 08-19 November 2021
}

(C) The Author(s), under exclusive licence to Springer Nature Switzerland AG 2022

\section{Pilot study of SOIE program for adjuvant endocrine therapy after breast cancer: a 3 months analysis}

\begin{abstract}
Assan O. ${ }^{1}$, Guillaumie L. ${ }^{2}$, Lemieux J. ${ }^{3}$, Provencher L. ${ }^{3}$, Gotay $\mathrm{C}^{4}{ }^{4}$, deBruin M. ${ }^{5}$, Lauzier $\mathrm{S}^{1}$

${ }^{1}$ Faculty of Pharmacy, Université Laval, Canada; ${ }^{2}$ Faculty of Nursing, Université Laval, Canada; ${ }^{3}$ Centre des maladies du sein, CHU de Québec-Université Laval, Canada; ${ }^{4}$ School of Population and Public Health, University of British Columbia, Canada; ${ }^{5}$ Radboud Institute for Health Sciences, The Netherlands (sophie.lauzier@pha.ulaval.ca)
\end{abstract}

Aim: Adjuvant endocrine therapy (AET) is prescribed for 5-10 years to women with hormone-sensitive breast cancer to reduce recurrence risks. Few AET adherence-enhancing interventions exist. We developed SOIE, a program including an educational group session and AET consultations. We pilot-tested the program by evaluating its effects on psychosocial factors hypothesized to influence AET adherence.

Methods: We conducted a pilot RCT among non-metastatic breast cancer women initiating AET. Psychosocial factors hypothesized to influence AET adherence were based on the theory of planned behavior (TPB, intention, attitude, subjective norm, behavioral control) and additional constructs (knowledge, social support, anticipated regret, coping planning) and were measured by questionnaires (baseline and 3-month follow-up). Group patterns were compared using repeated measures models with generalized estimating equation.

Results: 106 Women were randomized (intervention $\mathrm{n}=52$; control $\mathrm{n}=54)$. Both groups reported high levels on intention to adhere to AET (mean $\geq 6.0 / 7.0$ ). Patterns over time were not statistically different between groups for TPB constructs. Over time, the increase in knowledge and coping planning was greater in the intervention group than in control (difference of mean time differences: 1.23; p-value for group $*$ time interaction $=0.01$ and $0.48 ; \mathrm{p}=0.02$ ).

Discussion: The program has the potential to enhance women's AET knowledge and prepare them to cope with AET-related difficulties early in AET trajectory.

Conclusion: Maintenance of these early benefits and their role in preventing a decline in intention to adhere to AET as treatmentrelated difficulties evolve over time will be evaluated at 12 months.

\section{Management of medication adherence across ENABLE COST countries: a pilot study}

Sara Mucherino', Emma Aarnio ${ }^{2}$, Gaye Hafez ${ }^{3}$, Maria Kamusheva ${ }^{4}$, Francisca Leiva-Fernández, Jovan Mihajlović ${ }^{6,7,8}$, Miriam Qvarnström ${ }^{9}$, Ines Potočnjak ${ }^{10}$, on behalf of the ENABLE Project Group

${ }^{1}$ Center of of Drug Utilization and Pharmacoeconomics (CIRFF), Department of Pharmacy, Federico II University of Naples, Naples, Italy; ${ }^{2}$ School of Pharmacy, University of Eastern Finland, Kuopio, Finland; ${ }^{3}$ Department of Pharmacology, Faculty of Pharmacy, Altinbas University, Istanbul, Turkey; ${ }^{4}$ Department of Organization and Economics of Pharmacy, Faculty of Pharmacy, Medical University of Sofia, Bulgaria; ${ }^{5}$ Teaching Unit for Family and Community Primary Care Health District Málaga/Guadalhorce, Andalusian Health Service, Biomedical Research Institute of Málaga-IBIMA-, University of Malaga, Spain; ${ }^{6}$ Mihajlović Health Analytics, Novi Sad Serbia; ${ }^{7}$ University Medical Center Groningen, Groningen, The Netherlands; ${ }^{8}$ Medical Faculty, University of Novi Sad, Novi Sad, Serbia; ${ }^{9}$ Department of Pharmacy, Faculty of Pharmacy, Uppsala University, Uppsala, Sweden; ${ }^{10}$ Institute for Clinical Medical Research and Education, University Hospital Centre Sisters of Charity, Croatia (sara.mucherino@unina.it)

Introduction: The European Cooperation in Science and Technology (COST) Project "CA19132 - European Network to Advance Best practices \& technoLogy on medication adherencE" (ENABLE) Work Group 1 examines how countries manage medication adherence and the personal, healthcare professional (HCP), and health system barriers and facilitators of medication adherence.

Aim: To get knowledge on adherence-related practices and unmet needs perceived by different European HCPs.

Methods: Qualitative study with online survey sent to three key opinion leaders (KOLs) in five countries (Bulgaria, Croatia, Spain, Sweden, Turkey) involved in the ENABLE action. KOLs selected were HCPs and adherence experts from academia, hospital, and professional associations. Survey contained responders' background information and 12 open questions. Answers were analysed in three levels (patient, HCP, national/healthcare system) with Framework method through a coding process to establish thematic groupings.

Results: 12 KOLs responded. Most crucial patient-related barriers are absence of awareness, and need for training/education. The issues among HCPs are lack of awareness, data accessibility, need for 
training and guidelines, and insufficient collaboration between professionals. The most significant barriers related to healthcare systems are lack of legal basis, policies, national programs, accessible medication adherence technology, and digital solutions.

Conclusion: Lack of awareness and resources regarding medication adherence could be identified as the main barrier/unmet need in all three levels analysed in this pilot study. These findings will be confirmed in a survey to all 39 COST ENABLE countries.

\section{Results of the GAMER trial: gaming for adherence to medication using E-health in rheumatoid arthritis}

\section{B. P. H. Pouls ${ }^{1,2}$, C. L. Bekker ${ }^{2}$, J. E. Vriezekolk ${ }^{1}$,} S. van Dulmen ${ }^{3,4}$ and B. J. F. van den Bemt ${ }^{1,2}$

${ }^{1}$ Sint Maartenskliniek, Department of Rheumatology Research, Nijmegen, The Netherlands; ${ }^{2}$ Radboud University Medical Centre, Radboud Institute for Health Sciences, Department of Pharmacy, Nijmegen, The Netherlands; ${ }^{3}$ Nivel (Netherlands Institute for Health Services Research), Utrecht, The Netherlands; ${ }^{4}$ Radboud University Medical Centre, Radboud Institute for Health Sciences, Department of Primary and Community Care, Nijmegen, The Netherlands (b.pouls@maartenskliniek.nl)

Introduction: Effectiveness of pharmacological therapy in rheumatoid arthritis (RA) is limited by inadequate medication adherence. Medication adherence can be influenced by implicit (unconscious) judgements concerns about adverse consequences. We targeted these implicit judgments using a serious puzzle game.

Aim: To examine the effectiveness of a serious game to improve implementation adherence in patients with rheumatoid arthritis treated with anti-rheumatic drugs.

Methods: A multi-centre randomised clinical trial was performed with a 3 month follow-up period.*

Inclusion criteria were adulthood, rheumatoid arthritis, use of antirheumatic drugs and possession of a smartphone/tablet. Control patients received usual care whereas intervention patients were invited to install and play the serious puzzle game at will.

Primary outcome was proportion of adherent patients at 3 months as scored by the Compliance Questionnaire in Rheumatology. Additionally, beliefs about medication, Rheumatoid Arthritis Disease Activity Index, app usage data and app usability were collected.

Results: In total 187 patients completed the study. Of the 104 intervention patients, $93(89 \%)$ played the intervention. Median playtime was $444 \mathrm{~min}$ (IQR 156-1269 min). Usability score was 65 out of 100 . Adherence and disease activity data is currently being analysed and will be presented during the conference.

Discussion and conclusion: A serious puzzle game that targets implicit medication judgments was frequently played during a randomised intervention study. We are looking forward to present the data on its effectiveness during the conference.

*Netherlands Trial Register NL7217: https://www.trialregister.nl/ trial/7217.

\section{Motivational interviewing to support medication adherence in chronic conditions: systematic review of randomized controlled trials}

\author{
Teddy Novais ${ }^{1,2}$, Marlene Papus ${ }^{1}$, Marie Viprey ${ }^{2,3}$, \\ Anne-Marie Schott ${ }^{2,3}$, Marie P. Schneider ${ }^{4,5}$, Alexandra L. Dima ${ }^{2}$ \\ ${ }^{1}$ Service pharmaceutique, Hopital des Charpennes, Hospices Civils de \\ Lyon, Lyon, France; ${ }^{2}$ Université Claude Bernard Lyon 1, Research on
}

Healthcare Performance (RESHAPE), INSERM U1290, Lyon, France; ${ }^{3}$ Hospices Civils de Lyon, Pôle de Sante Publique, Lyon, France; ${ }^{4}$ Chair of Medication Adherence and Interprofessionality, School of Pharmaceutical Sciences, University of Geneva, Geneva, Switzerland; ${ }^{5}$ Institute of Pharmaceutical Sciences of Western Switzerland, University of Geneva, Switzerland (teddy.novais@chulyon.fr)

Introduction: Motivational interviewing (MI) is increasingly used to support medication adherence, and research on its efficacy is accumulating.

Aim: To systematically review published randomized controlled trials (RCTs) assessing the efficacy of MI to support medication adherence in adults with chronic conditions.

Methods: A systematic review (PROSPERO-CRD42020025374) was performed by searching in PubMed/MEDLINE, PsycINFO, The Cochrane Library and Web of Science. Two reviewers conducted independent screening of records and full-text articles published until July 2020. Quality was assessed with the Risk of Bias 2 tool for RCTs.

Results: From 1262 studies identified, 55 RCTs were included (17,405 patients). The MI interventions were delivered alone or in combination with other interventions, and varied in mode of delivery (e.g. face-to-face, phone), exposure level (duration, number of sessions), and provider characteristics (profession, training). Most interventions were developed in infectiology $(n=16)$, cardiology $(n=14)$, psychiatry $(n=8)$, and endocrinology $(n=7)$. Medication adherence showed significant improvement in 23 RCTs, and other clinical outcomes were improved in 19 RCTs (e.g. risky behaviors, disease symptoms).

Discussion: Two prior meta-analyses included 25 studies published until 2015 (mainly in HIV, 12/25). Our review identified 26 additional RCTs published since. While 23 studies showed promising results, further research should focus on improving study quality and comparability, and consider less explored diseases or populations.

Conclusion: $\mathrm{MI}$ is an approach to medication adherence support with an increasing evidence base in several clinical domains and further potential for adaptation to different settings.

\section{Exploring stem cell transplant patients' acceptability and preferences of three medication adherence electronic monitoring systems}

Ribaut J. ${ }^{1,2}$, De Geest $S^{1,3}$, Leppla L. ${ }^{1,4}$, Gerull S. ${ }^{2,5}$, Teynor A. ${ }^{6}$, Valenta $S .{ }^{1,2}$, on behalf of the SMILe Study Team

${ }^{1}$ Institute of Nursing Science, Department Public Health, Faculty of Medicine, University of Basel, Switzerland; ${ }^{2}$ Department of Theragnostik, Haematology, University Hospital of Basel, Switzerland; ${ }^{3}$ Academic Center for Nursing and Midwifery, Department of Public Health and Primary Care, KU Leuven, Belgium; ${ }^{4}$ Departments of Hematology, Oncology and Stem Cell Transplantation, University Medical Center Freiburg, Germany; ${ }^{5}$ Department of Hematology, Cantonal Hospital of Aarau, Switzerland; ${ }^{6}$ Department of Computer Science, University of Applied Sciences, Augsburg, Germany (janette.ribaut@unibas.ch)

Introduction: Acceptability and preferences of electronic monitoring (EM) systems assessing medication adherence (i.e., initiation, implementation, persistence) are crucial for a successful implementation in clinical or research environments. Various EM systems exist, yet patient acceptance and preferences have not yet been evaluated. Aim: We aimed to assess adult allogeneic stem cell transplant patients' (1) medication adherence strategies; and (2) acceptability 
and preferences of 3 EM systems (MEMS ${ }^{\circledR}$ Cap, Helping Hand, Button).

Methods: We conducted three focus groups with a purposive sample of six patients $(3 \times 2$ patients due to COVID-restrictions, 6 weeks -2 years post-transplant). Using a semi-structured interview guide, we explored patients' (1) medication adherence strategies; and (2) acceptance and preferences on EM use. All EM systems were presented and open for testing during the focus groups. Discussions were audio-recorded, summarized in a mind-map, and analyzed using Mayring's qualitative content analysis.

Results: Patients (33\% females; mean age 54.6 $\pm 16.3 ; 10.4 \pm 8.4$ months post-transplant) reported own medication adherence enhancing strategies (e.g., preparing pillbox) yet that medication management was challenging (e.g., frequent schedule changes). All participants preferred the MEMS ${ }^{\circledR}$ Button because it is small and can be combined with existing pillboxes and felt confident implementing the MEMS ${ }^{\circledR}$ Button in daily life.

Conclusion: We found the highest acceptability and preference for the MEMS ${ }^{\circ}$ Button and will therefore use it in our study. Shedding light on acceptability and preference of EM systems is crucial to maximize adoption and fidelity with these systems in trial and clinical settings.

\section{Adherence to medications and government recommendations among breast cancer women during the COVID-19 pandemic}

\section{Lauzier S. ${ }^{1}$, Bourgault F. ${ }^{1}$, Dorval M. ${ }^{1}$, Nabi H. ${ }^{4}$, Savard J. ${ }^{3}$, Bennett K., Myers C., Cahir C. ${ }^{2}$}

${ }^{1}$ Faculty of Pharmacy, Université Laval, Canada; ${ }^{2}$ Royal College of Surgeons, Ireland; ${ }^{3}$ School of Psychology, Université Laval, Canada, RCSI Dublin, Ireland (caitrionacahir@rcsi.ie); ${ }^{4}$ Faculty of Medicine, Université Laval, Canada

Aim: The COVID-19 pandemic is affecting cancer and supportive care for women diagnosed with breast cancer and various aspects of their lives. In this context, we sought to document adherence to oral breast cancer medications and adherence to government measures related to the pandemic.

Methods: We conducted an online survey between 2020/11 and 2021/03 in the Province of Quebec (Canada) and Ireland among women diagnosed with breast cancer in the past 5 years. Beliefs regarding oral breast cancer medications and adherence were measured using the Belief about medicines questionnaire (BMQ) and the Medication adherence report scale (MARS). These instruments were adapted to assess beliefs and adherence to government measures related to the pandemic.

Results: 650 Women responded to the online survey (Quebec, $n=265$; Ireland, $\mathrm{n}=385) .385(59 \%) \mathrm{Had}$ oral medications at time of the survey (97\% hormone therapy). Respondents had positive beliefs about oral breast cancer medications (mean difference between BMQ necessity and concerns scores: Quebec +5.6 ; Ireland +7.3) and high adherence (mean 24/25 for both settings). Positive beliefs were also reported for recommendations regarding social distancing $(+6.28$; $+5.36)$ and mask wearing $(+8.59$; +12.18$)$. Adherence to government recommendations was also high in both settings $(22 / 25)$.

Discussion: Women with breast cancer reported positive beliefs and very high adherence to both oral medications and government recommendations.

Conclusion: An undergoing qualitative study will explore the reasons for high adherence among this vulnerable population facing pandemic challenges.
Medication adherence and healthcare costs related to multiple sclerosis treatment

Eugenio Di Brino ${ }^{1}$, Michele Basile ${ }^{1}$, Filippo Rumi ${ }^{1}$, Ilaria Guarino $^{2}$, Sara Mucherino ${ }^{2}$, Valentina Orlando ${ }^{2}$, Enrica Menditto ${ }^{2}$, Americo Cicchetti ${ }^{1}$

${ }^{1}$ Graduate School of Health Economics and Management, Università Cattolica del Sacro Cuore, Rome, Italy; ${ }^{2}$ Center of Drug Utilization and Pharmacoeconomics (CIRFF), Department of Pharmacy, Federico II University of Naples, Naples, Italy (eugenio.dibrino@unicatt.it)

Introduction: Level of adherence to multiple sclerosis (MS) treatments, are often inadequate with rates ranging from 60 to $85 \%$. This leads to increased morbidity and mortality as well as health care costs. Aim: To evaluate adherence to treatment of patients with MS and related healthcare costs.

Methods: Retrospective cohort study was carried out in Campania region (southern Italy). All subjects with at least one prescription of drugs for MS treatment and/or an hospital discharge for MS, were included in the analysis. Second phase of the adherence process, the implementation, was assessed by calculating medication possession ratio (MPR). Hence, as per ESPACOMP recommendations, MPR was expressed as a percentage and truncated to 100 when $>100 \%$. Implementation was considered suboptimal were MPR $\leq 80 \%$ (nonadherent). Analysis of hospitalizations costs was stratified by adherent and non-adherent subjects. This work was supported by an unrestricted educational grant from Merck.

Results: 4575 were patients suffering from MS, of which $24.5 \%$ were non-adherent to treatment (MPR $\leq 80 \%$ ). Overall hospitalization rate was $78 \%$. Non-adherent patients recorded higher percentage of hospitalization for MS cause versus adherent patients $(81.1 \%$ vs $77 \%)$. Cost analysis revealed that average cost per hospitalization was higher in non-adherent patients ( $€ 1909$ vs. $€ 1830$ respectively).

Conclusion: Findings revealed a correlation between non-adherence and increased risk of hospitalization with consequent increase in healthcare costs. Improving adherence remain a priority for health systems in terms of both health outcomes and healthcare costs.

\section{Gender differences in medication adherence among adolescent and young adult solid organ transplant recipients}

\section{Vaisbourd Y. ${ }^{1}$, Dahhou M. ${ }^{1}$, Foster B. J. ${ }^{1}$, Vaisbourd $^{1}$ \\ ${ }^{1}$ McGill University, Canada (yulia.vaisbourd@mail.mcgill.ca)}

Aim: To compare the implementation phase of immunosuppressive medication adherence between male and female transplant recipients. Methods: This Canadian multicenter prospective observational cohort study of prevalent kidney, liver, and heart transplant recipients 14-25 years old assessed adherence at 0,3 , and 6 months using the BAASIS self-report tool. At each visit, participants were classified as adherent if they missed no doses in the prior 4 weeks. Standard deviation (SD) of tacrolimus trough levels (from 6 months before to 6 months after the first visit) was assessed as a secondary outcome; a SD $<2.0$ was classified as adherent. We used multivariable mixed effects logistic regression accounting for repeated measures within participants and clustering by program. Models were adjusted for parent vs. self as caregiver, caregiver education, insurer and time from transplantation.

Results: At baseline, $69 \%$ of the 180 males and $74 \%$ of the 120 females were adherent based on self-report, whereas $66 \%$ of males 
and $56 \%$ of females were adherent based on tacrolimus SD. Males were significantly less likely to be adherent than females based on self-report (OR $0.4,95 \%$ CI $0.2-0.8$ ) but more adherent based on tacrolimus SD (OR 2.2, 95\% CI 1.2-3.9).

Discussion: These findings are consistent with those of a prior study which showed poorer electronically-monitored adherence in males than females, but better adherence based on tacrolimus SD.

Conclusion: Females have better adherence than males. Factors other than medication adherence may influence SD of tacrolimus levels.

\section{Differences in glaucoma perception and medication adherence among patients of African and European descent}

\author{
Poleon S. ${ }^{1}$, Abu S. ${ }^{1}$, Thomas T. ${ }^{1}$, Racette L. ${ }^{1}$ \\ ${ }^{1}$ University of Alabama at Birmingham, USA (skpoleon@gmail.com)
}

Aim: Patients of African descent (AD) often have worse adherence than patients of European descent (ED) during the implementation phase of adherence. We aimed to identify whether this was associated with differences in glaucoma perception and whether medication adherence was associated with glaucoma perception.

Methods: We measured adherence in $51 \mathrm{AD}$ and $56 \mathrm{ED}$ patients with Medication Event Monitoring Systems. Perceptions about glaucoma were assessed using the brief illness perception questionnaire (BIPQ). We conducted Mann-Whitney U test for racial differences in overall BIPQ score and multiple linear regression to assess the relationship between overall BIPQ score and mean adherence.

Results: AD patients had lower mean adherence $(74.9 \% \pm 22 \%$ vs. $88.7 \% \pm 16 \% ; \mathrm{p}<0.001)$ than ED patients. There were no significant racial differences in overall BIPQ score or significant relationships between mean adherence and overall BIPQ score. However, AD patients had higher BIPQ scores for the timeline subscale, which measures perceived glaucoma duration $(\mathrm{p}=0.01)$ and for the concern subscale, which measures perceived glaucoma severity $(\mathrm{p}=0.049)$.

Discussion: AD patients are more likely to be younger and to have more advanced glaucoma at the time of diagnosis. This may be cognitively represented as we found that $\mathrm{AD}$ patients had higher perceived glaucoma duration and higher perceived glaucoma severity. Conclusion: AD patients had worse adherence, higher perceived glaucoma duration and higher perceived glaucoma severity. By incorporating racial differences in glaucoma perception, interventions for improving adherence can.

\section{Comparison of different methods to assess tacrolimus intra-patient variability and its relation to medication adherence}

\author{
Kostalova B. ${ }^{1}$, Mala-Ladova K. ${ }^{1}$, Denhaerynck K. ${ }^{2}$, \\ Dusilova Sulkova $\mathrm{S}^{3}{ }^{\mathbf{3}}$, Maly $\mathrm{J}^{\mathbf{1}}$ \\ ${ }^{1}$ Department of Social and Clinical Pharmacy, Faculty of Pharmacy in \\ Hradec Kralove, Charles University, Czech Republic, Dedication: \\ SVV $260551 ;{ }^{2}$ Institute of Nursing Science, Department Public \\ Health, University of Basel, Switzerland; ${ }^{3}$ Haemodialysis Center, \\ University Hospital Hradec Kralove, Czech Republic \\ (vankovb@faf.cuni.cz)
}

Aim: High tacrolimus intra-patient variability (IPV) indicates patient's exposition to both high and low drug levels, while low levels are mostly associated with medication non-adherence. We aimed to compare different methods of tacrolimus IPV calculations and evaluate, whether the superior IPV method combined with self-report can improve the identification of kidney transplant recipients at risk of implementation phase non-adherence.

Methods: This Czech unicentric retrospective study was conducted in 2019. All outpatients $\geq 18$ years of age, $\geq 3$ months posttransplant, and on tacrolimus were approached. Using regression analysis, nonadherence to immunosuppressants measured by the self-report BAASIS $\odot$ questionnaire, predicted IPV calculated from seven consecutive tacrolimus concentrations. IPV was operationalized in four ways, i.e. as medication level variability index (MLVI), coefficient of variance $(\mathrm{CV})$, time-weighted $\mathrm{CV}$, and using nonlinearly modeled dose-corrected trough levels.

Results: Out of 243 included patients, self-reported implementation phase non-adherence during the last 4 weeks was admitted by 102 patients. Non-adherence was associated with higher $\mathrm{CV}$, higher timeweighted $\mathrm{CV}$ and lower dose-corrected nonlinearly modeled trough levels, but not with MLVI. All of the significant operationalizations suggested a weak relationship, similar across the applied methods.

Discussion and conclusion: Simple calculation of $\mathrm{CV}$ did not underperform as compared to a more sophisticated nonlinear regression analysis.

\section{Medication adherence in registration trials supporting the approval of new medicines in the European Union}

Katerina M. Mantila ${ }^{1,2,3}$; Peter G. M. Mol ${ }^{2,3}$; Marjon Pasmooij ${ }^{3}$; Christine Erikstrup Hallgreen ${ }^{1}$; Job F. M. van Boven ${ }^{2,4}$

${ }^{1}$ Copenhagen Centre for Regulatory Science, Department of Pharmacy/Pharmaceutical Sciences, University of Copenhagen, Denmark; ${ }^{2}$ University of Groningen, University Medical Center Groningen, Department of Clinical Pharmacy \& Pharmacology, Groningen, The Netherlands; ${ }^{3}$ The Dutch Medicines Evaluation Board-College ter Beoordeling van Geneesmiddelen, Utrecht, The Netherlands; ${ }^{4} \mathrm{Med}-$ ication Adherence Expertise Center of the Northern Netherlands (MAECON), Groningen, The Netherlands (katerinamantila@gmail.com)

Introduction and aim: Medication adherence is one of the factors impacting drug efficacy in registration trials. We aimed to assess how medication adherence (both implementation and persistence) is addressed in registration studies for new medicines in Europe.

Methods: A quantitative cross-sectional analysis of European Medicines Agency (EMA) marketing authorisation dossiers for new medicines approved through centralised procedures in the EU between 2010 and 2020 was performed. Data were extracted from EPARs and from Clinical Study Reports. Pivotal efficacy studies in these dossiers were selected for five therapeutic areas: respiratory conditions, diabetes, oncology, cardiovascular diseases and anti-infectives. Treatments with indicated use period of at least 3 months to lifelong (chronic) were included. Outcomes included adherence measurement, method, and rates.

Results: For this interim report (date: 11/6/2021; anticipated full analysis completed: 29/8/2021), 89 medicines and 206 pivotal efficacy studies were reviewed. $98 \%$ of the trials measured adherence. The most common measurement method used was pill count, used by $80 \%$ of the trials. Other main methods used were patient diary/report forms $(15 \%)$ and questionnaires (1\%). 72 Studies used multiple methods to verify adherence (including blood samples). Overall adherence rates were over $90 \%$.

Discussion: Overall, adherence is measured in EMA registration trials, yet the methods used and the way in which adherence rates are presented varies between trials. 
Conclusion: The way adherence is addressed in EMA registration trials varies between medicinal products. More standardization on definitions and measurement methods seems required.

\section{Mapping the cardiometabolic patient experience to inform initiation, implementation and persistent use of virtual healthcare}

\author{
Liska J. ${ }^{\mathbf{1}}$, Mical M. ${ }^{1}$, Maillard C. ${ }^{\mathbf{1}}$, Dessapt-Baradez C. ${ }^{\mathbf{1}}$, \\ Bendig E. ${ }^{2}$, Mai D. ${ }^{2}$, Fontaine G. ${ }^{3}$ \\ ${ }^{1}$ Sanofi, France $;{ }^{2}$ Sturm und Drang, Germany; ${ }^{3}$ Ottawa Hospital \\ Research Institute, Canada (jan.liska@sanofi.com)
}

\begin{abstract}
Aim: This survey for cardiometabolic patients diagnosed with T2 diabetes and/or an ACS aims at providing deep human-centric insights into the behavioral dimension of their patient experience, while mapping future-focused opportunities to increase adherence for chronic and acute patients, facilitate healthy behaviors, and improve patient outcomes.

Methods: We employed virtual observational ethnography-a socialscientific methodology of qualitative research-to detect sociocultural patterns and differences among a sample of patients from US, Germany and Taiwan, composed by $50 \%$ compliant and $50 \%$ noncompliant patients. 98 Online auto-ethnographic diaries and 48 semistructured web-based interviews patients and caregivers were conducted to understand self-care adherence drivers from medical, psychological and cultural perspectives.

Results: Cardiometabolic patients see their journeys as non-linear, influenced by cognitive and/or emotional aspects of their health and life continuous changes. Adherence to care interventions appears as result of an ongoing process. Self-care initiation and -maturity in cardiometabolic patients evolve with their mind state. Tailored support considering patients' current mind state may result in optimal initiation, adherence and self-care expertise.

Discussion: Providers need to assess patients' mind state prior to care initiation for optimal patient engagement. Sustained virtual care solutions that help patients step up gradually from struggling or juggling to controlling mind state and embrace fully patient-relevant social determinants of health, are likely to increase patient self-care expertise.
\end{abstract}

Conclusion: This survey opens novel perspectives for disease management design, implementation and patient adherence monitoring.

\section{Managing medications during the early phases of the COVID-19 pandemic}

Bartlett Ellis, R. J. ${ }^{1}$, Ruggeri, S. Y. ${ }^{2}$, Ruppar, T. M. ${ }^{3}$, Russell Lippincott, C. L. ${ }^{2}$, Andrews, A. ${ }^{4}$, Remy, L. ${ }^{5}$, and Elomba, C. D. ${ }^{1}$

${ }^{1}$ Indiana University, United States; ${ }^{2}$ University of Missouri-Kansas City, United States; ${ }^{3}$ Rush University, United States; ${ }^{4}$ Southern Illinois University-Edwardsville, United States; ${ }^{5}$ Stowers Institute for Medical Research, United States (rjbartle@iu.edu)

Aim: To explore medication management and adherence, including implementation and persistence, during the early phases of the COVID-19 pandemic.

Methods: A descriptive survey was delivered via social media between May and July 2020. Adherence was measured by the self- report BAASIS $\subset$ scale and other items to assess the pandemic's impact on interactions with providers and pharmacies.

Results: A total of 134 respondents (mean age 50.0; $79.1 \%$ female; $77.6 \%$ white) reported taking at least one prescribed medication daily. Respondents took 3.45 mean (SD 2.68) daily medications. New prescriptions or refills were reported by $77.6 \%$, of which $18.7 \%$ reported having to change methods for obtaining medications due to the pandemic. Comments indicated that drive-thru options and mail order services helped with obtaining medication; however, $4.5 \%$ of the sample reported they stopped taking medications during the pandemic due to inability to refill medications. $38.2 \%$ of respondents reported missing medication doses within the last 4 weeks. 67\% Reported that taking medications was part of their routines; however, $3.4 \%$ reported declines in routines during the pandemic. Additionally, $37.5 \%$ delayed seeing a provider; $35 \%$ reported the delay was due to fear of leaving the house.

Discussion: During a pandemic, interventions to support medicationtaking in the absence of usual daily routines become increasingly important.

Conclusion: Processes to get medications to patients that do not require physical interaction with other people (risk of exposure) are particularly important, as well as.

\section{Patients experiences about real-life electronic monitoring of DOAC adherence in community pharmacies}

Desmaele Sara ${ }^{1}$, Vrijens Bernard ${ }^{2}$, Boussery Koen ${ }^{3}$, Capiau Andreas ${ }^{3}$, Grymonprez Maxim ${ }^{3}$, Lahousse Lies ${ }^{3}$, Mehuys Els ${ }^{3}$, Steurbaut Stephane ${ }^{4}$, Rydant Silas ${ }^{1,4,5}$

${ }^{1}$ Koninklijke Apothekersvereniging Antwerpen (KAVA), Belgium; ${ }^{2}$ Vrijens Bernard, AARDEX GROUP \& Liège University, ${ }^{3}$ Pharmaceutical Care Unit, Faculty of Pharmaceutical Sciences, Ghent University, Belgium; ${ }^{4}$ Research Group of Clinical Pharmacology and Clinical Pharmacy, Vrije Universiteit Brussel/UZ Brussel, Belgium; ${ }^{5}$ Laboratory of Physiopharmacology, Department of Pharmaceutical Sciences, University of Antwerp (silas.rydant@kava.be)

Adherence to direct oral anticoagulants (DOAC) remains suboptimal. Electronic monitoring systems are ideal to follow up adherence, however not routinely implemented in daily practice. This study assesses the implementation of Medication Event Monitoring systems (MEMS $\left.{ }^{\circ}\right)$ in community pharmacies. Patients on DOAC were recruited consecutively in 13 Belgian community pharmacies and received a MEMS ${ }^{\circledR}$-cap for a first period of 6 weeks. After this period, pharmacists read out the MEMS $\AA$-cap and discussed the results with the patients. Subsequently, the MEMS $₫$-cap was refilled for another 6 weeks. Participants completed a survey before the start of the study and after $6 / 12$ weeks. A total of 158 patients were addressed, of which $56.0 \%$ agreed to participate. The most common reason to refuse was the actual use of another medication box $(43.5 \%)$. A quarter of the patients believed that their adherence increased during the study period, mostly due to the combination of electronic monitoring and interventions by the pharmacist. Although most patients $(62.5 \%)$ believed that adherence is their responsibility, the majority $(55.0 \%)$ was grateful for the coaching by their pharmacist and/or general physician. About $80 \%$ of the patients gained more insight in their medication use. Despite the limited number of participants, this study demonstrates the feasibility of electronic adherence monitoring in community pharmacies. Patients were satisfied with the service and reported a better insight in their medication taking behaviour as well as an increased adherence in about $25 \%$ of cases. 
The perspective of breast cancer women participating in an adherence-enhancing program for adjuvant endocrine therapy

\section{Turcotte V. ${ }^{1}$, Guillaumie L. ${ }^{2}$, Lemay $\mathrm{M.}^{3}$, Dionne A. ${ }^{4}$,} Lemieux J. ${ }^{3}$, Provencher L. ${ }^{3}$, Lauzier S.

${ }^{1} \mathrm{CHU}$ de Québec-Université Laval Research Center, Canada; ${ }^{2} \mathrm{Fac}-$ ulty of Nursing, Université Laval, Canada; ${ }^{3}$ Centre des maladies du sein, CHU de Québec-Université Laval, Canada; ${ }^{4}$ Faculty of Pharmacy, Université Laval, Canada (sophie.lauzier@pha.ulaval.ca)

Aim: Daily adjuvant endocrine therapy (AET) is prescribed for 5-10 years to women with hormone-sensitive breast cancer to prevent recurrence. We developed SOIE (Soutien, Outils, Information, Entraide), a 1-year program aimed at enhancing the experience with AET and treatment adherence. The program comprises an educational group session, individual AET consultations and online chat sessions. The program was pilot-tested in a mixed-methods RCT. We report here on women's perceived impacts of this program.

Methods: We conducted a qualitative study among 20 women who participated in the program. Participants were interviewed about their experiences using a semi-structured interview guide at the end of the 1-year follow-up. Interviews were audio-taped, transcribed verbatim, and analyzed using thematic analysis.

Results: Main perceived benefits of the program were: (1) fostering the understanding of the importance of AET; (2) learning strategies to cope with side effects; (3) having better access to services related to AET; (4) normalizing the experience with AET; and (5) reducing the feeling of loneliness. Participants also provided several suggestions to improve the program.

Discussion: Findings suggest that SOIE program can enhance the experience of AET by meeting important needs related to understanding the role of AET, coping with AET-related difficulties and feeling supported. However, translation of these benefits into enhanced adherence was more rarely explicitly reported.

Conclusion: SOIE can better equip women to cope with AET and enhance their well-being during this essential phase of breast cancer treatment.

\section{Medication self-management in psychiatry: Healthcare providers' and patients' perspectives}

\author{
Loots, Elke ${ }^{1}$ \\ ${ }^{1}$ University of Antwerp, Belgium (elke.loots@uantwerpen.be)
}

Background: Medication self-management concerns the process in which hospitalised patients instead of healthcare providers prepare and consume medications by themselves. At the moment it is unclear how different aspects around this topic are managed in psychiatric hospitals and factors that promote or prevent this implementation. It is necessary to have insight into the perspectives of all stakeholders involved in order to implement medication self-management in psychiatric hospitals. The aim of the study was to explore perspectives of hospitalised patients with schizophrenia or a bipolar disorder and healthcare providers on medication self-management during hospitalisation.

Methods: A qualitative descriptive design using semi-structured interviews was adopted. Patients and healthcare providers were asked about their perspectives concerning medication self-management during hospitalisation in a face-to-face interview. All interviews were audio-recorded, transcribed and analysed in conformity with the principles of qualitative content analysis.
Results: Patients and nurses from one regional psychiatric hospital were interviewed. Patients and nurses indicated that insufficient knowledge of their medication is an obstacle. Patients need follow-up and extra support during this process. Patients and nurses reported education concerning medication should be a prerequisite for medication self-management during hospitalisation. The majority of nurses considered misuse of medication a disadvantage and unsafe.

Discussion: This study yielded new insights from the perspectives of key stakeholders concerning medication self-management during hospitalisation in psychiatry. These findings will be used in the development and implementation of a medication self-management tool in psychiatry.

\section{State of the art about the inclusion of medication adherence implications in pharmacoeconomic evaluations}

\author{
Salvatore Pirri ${ }^{1}$, Valentina Lorenzoni ${ }^{1}$, Giuseppe Turchetti $^{1}$ \\ ${ }^{1}$ Scuola Superiore Sant'Anna di Pisa, Italy (salvatore.pirri@ santannapisa.it)
}

Introduction: Pharmacoeconomic goal is to set the balance between cost and benefit of a treatment. Low medication adherence has high impact on the clinical effectiveness and can substantially change the cost estimate with the possibility of distorting the cost-effectiveness ratio of alternatives.

Aim: The aim of the research is to collect and review studies that consider medication adherence implications in pharmacoeconomic evaluations.

Methods: A literature review methodology was used, searching published articles in PubMed from 2015 to 2020 in English. Articles were included if they apply health economic evaluation related to somewhat medication adherence measures. The Medication Adherence Reporting Guideline (EMERGE) was used to verify the alignment with the ABC Taxonomy, and the CHEERS guidelines for the publication's quality assessment.

Results: A total of 89 articles were retrieved from PubMed, of which 21 were selected after screening the abstracts, and 6 were considered in this study. All studies were trials and employed cost-effectiveness analysis. Four out of six studies reported the intervention as costeffective.

Discussion: Non-adherence reduces the treatment effectiveness and negatively influences cost estimation, leading to inaccurate cost-effectiveness assessment. None of the studies reported the phases of medication adherence, and considered adherence phases implication in cost analysis.

Conclusion: Despite the well-recognised negative implications, no standard procedures are in place to integrate adherence rate in economic evaluations, nor the adherence state phases were properly reported.

\section{The HealthBeacon Injection Care Management System shows improved adherence to gastrological, dermatological and rheumatological treatments}

\author{
Sarhan, S. McWhinney ${ }^{1}$, S. Shah ${ }^{1}$, A. L. Kelly ${ }^{1}$ \\ ${ }^{1}$ HealthBeacon Limited, Dublin, Ireland (sharifah.sarhan@healthbeacon. \\ com)
}


Introduction: Treatment adherence falls sharply following initiation, and is reported to range from 46 to $66 \%$ during implementation, with variability by age, gender and therapeutic area.

Aims: By enhancing patient support and accommodating routines, HealthBeacon aims to improve adherence. We investigated its efficacy and compared outcomes with reported adherence.

Methods: HealthBeacon electronically monitored injections during 2015-2021 to calculate adherence and create a medication possession ratio (MPR) proxy of adherence $\geq 80 \%$, in 9737 patients $(55 \%$ female) ranging from 18 to 100 years of age. Regression identified effects of age, gender and therapeutic area. Results were compared with literature to identify benefits of the HealthBeacon ICMS.

Results and discussion: HealthBeacon's adherence was strong overall $(\mathrm{MPR}=0.865, \mathrm{SD}=0.037)$. Regression showed that adherence increased significantly with age while controlling for other effects $[\mathrm{F}(4,22)=7.47, \mathrm{p}<0.001]$, with post hoc testing showing adherence as significantly highest in the oldest age group (MPR = $0.913, \mathrm{SD}=0.054)$. Previously published differences in gender and therapeutic area in the literature were not found in HealthBeacon's data. For those on rheumatological treatments, the proportion of HealthBeacon patients with MPR $\geq 0.80(66 \%)$ significantly exceeded the $95 \%$ confidence interval of reported studies (45-62\%). MPR was also high for gastrological $(\mathrm{MPR}=0.860, \mathrm{SD}=0.030)$ and dermatological (MPR $=0.870, \mathrm{SD}=0.050)$ treatments, comparing favourably with previous reports in each $(62 \%$ and $61 \%)$.

Conclusion: HealthBeacon's support resulted in significantly higher adherence than other reports, mirroring age effects, and potentially mitigating gender differences. This system represents a significant step in improved adherence for several therapeutic areas.

\section{Patients' opinions on hypertension and antihypertensive drugs: a qualitative study}

\author{
Malkon S. ${ }^{1}$, Wettermark B. ${ }^{1}$, Qvarnström M. ${ }^{1}$ \\ ${ }^{1}$ Uppsala University, Sweden (miriam.qvarnstrom@farmaci.uu.se)
}

\begin{abstract}
Aim: To gain an understanding of patients' opinions about- and experiences of hypertension and the use of antihypertensive drugs.

Methods: A qualitative study based on open-ended questions from a previous survey on medication adherence, that captured hypertension patients' perspective on their condition and antihypertensive treatment. Data were collected during 2016 for patients $\geq 30$ years old consulting 25 different primary health centers in the southwestern part of Stockholm County, Sweden. Thematic analysis with both inductive and deductive approach was applied.
\end{abstract}

Results: A total of 219 patients with hypertension were included. We identified 21 codes from the data and grouped them under the World Health Organization's five dimensions of adherence: condition-, therapy-, health care team and system-, patient-, and socioeconomicrelated factors, that were used as themes in the thematic analysis. Patients with hypertension feel that they have limited knowledge of hypertension, are afraid of drug side effects and they experience various issues in primary care: short doctor appointments, prescribing without communication and room for improvement in individualization of therapy and a person-centered approach.

Discussion: The factors mentioned among the questionnaire responses could affect all three phases of medication adherence: initiation, implementation and persistence. The most important strength of the study is the varied population, while sampling- and nonresponse bias are the major limitations.

Conclusions: Patients with hypertension raise several problems related to the consultation and drug prescribing.
Digital-storytelling in health context: innovative approaches to improve patients and caregivers' therapeutic literacy and adherence

\author{
Sabrina Grigolo ${ }^{1}$; Manuela Repetto ${ }^{2}$; Melania Talarico $^{3}$; \\ Simona Tirocchi ${ }^{4}$; Sara Croce ${ }^{5}$; Clara Cena ${ }^{6}$; \\ Lisa Iannacci-Manasia ${ }^{7}$; Barbara Bruschi ${ }^{8}$
}

${ }^{1}$ EPALE Ambassador, Expert Patient EUPATI, Adjunct Professor of Therapeutic Education and Chronic Nursing, $\mathrm{PhD}$ Student on Department of Philosophy and Education Science and Department of Drug Science and Technology of Turin University (IT); ${ }^{2} \mathrm{PhD}$, Researcher, University of Turin; ${ }^{3} \mathrm{PhD}$, Research Fellow of Department of Philosophy and Education Sciences, University of Turin; ${ }^{4}$ Associate Professor in Sociology of culture and communication; ${ }^{5}$ Student of Nursing School, University of Turin; ${ }^{6}$ Associate Professor in Medicinal Chemistry at the Drug Science and Technology Department ("Dipartimento di Scienza e Tecnologia del Farmaco", DSTF) in Turin; ${ }^{7}$ MS, PNP, Faculty, Academic Affairs Division, Columbia University School of Nursing; ${ }^{8}$ Professor of Pedagogy and Media digital Storytelling, University of Turin (sabrina.grigolo@unito.it)

Introduction: It is well documented in the literature with studies of overall adherence rates estimated to be $50 \%$ for the majority of longterm therapy and treatments for chronic disease. While many studies focus on patients adherence to specific treatments for specific conditions, very few studies examine the concept of adherence from the patient's perspective i.e. the lived experience of adhering to complex therapy express by the patient. Advances in multimedia technology have allowed opportunities for the use of digital storytelling as an intervention for health-related outcomes.

Aims: To explore factors that impact adherence to therapy and to identify educational and digital strategies to enhance patient self awareness levels. To develop and validate a measurement tool for assessing patient skills.

Methods: A mixed-methods multicenter study has been implemented in the Piedmont Region Health System.

Results: Validation of the assessment tool, as well as evaluation of patient therapeutic adherence skills and patient digital knowledge and educational preferences are ongoing. The impact of digital storytelling on health literacy will be evaluated in a second phase of the study.

Discussion and conclusion: Digital questionnaires will be tested and educational preferences as well as therapeutic adherence skills will be assessed.

The assessment test, once validated, represents a tool to detect patients' skills on therapeutic adherence. Moreover, the experimentation of the digital storytelling approach on the selected sample of patients will inform the scientific community about the effectiveness of this method on the improvement health literacy.

\section{Acceptance and adherence to COVID-19 vaccine in a Portuguese Population Survey}

\author{
Sampaio, R. ${ }^{1}$, Cruz, M. ${ }^{1}$, Pinto, S. ${ }^{1}$, Dias, C. ${ }^{1}$ and Lopes, J. C. ${ }^{1}$ \\ ${ }^{1}$ Portugal (rutesampaio@med.up.pt)
}

Aim: The recognition of the phenomenon of vaccine hesitation is of utmost importance mainly during the current pandemic. Herein, we aim at evaluating COVID-19 vaccine acceptance in the Portuguese population and understand the cognitive and emotional representation of COVID-19 and its vaccine. 
Methods: A cross-sectional online survey was conducted between 27th December 2020 and 27th January 2021. The questionnaire assessed: cognitive and emotional COVID-19 representations; COVID-19 vaccine status; cognitive and emotional representations of the vaccination; and the perceived necessity and perceived concerns about the COVID-19 vaccine.

Results: Of 3158 participants, 91\% accepted COVID-19 vaccine. Compared with men, women $(71.3 \%)$ considered that the pandemic has affected more their lives $(\mathrm{p}<0.001)$, are more concerned with being infected $(\mathrm{p}<0.001)$ and feel more emotionally disturbed by the pandemic situation $(p<0.001)$. It is important to highlight that perceiving itself as extremely informed about the infection does not provide for acceptance of the vaccine $(\mathrm{OR}=1.534$ [1.160-2.029]; $\mathrm{p}=0.003$ ). Age groups between 25 and 65 years and with lower education level are at higher risk of not accepting the vaccine $(\mathrm{OR}=2.799$ [1.085-7.221]; $\mathrm{p}=0.033)$. Being more concerned about taking the vaccine decreases its acceptance $(\mathrm{OR}=4.001$ [2.518-6.356]; $\mathrm{p}<0.001$ ).

Conclusion: It is very important that public health messages are adapted to the different characteristics of the population.

\section{Adherence behaviour change during COVID-19 outbreak in Portugal}

\begin{abstract}
Midão L. ${ }^{1,2}$, Almada M. ${ }^{1}$, Sampaio R. ${ }^{2}$, Costa E. ${ }^{1}$
${ }^{1}$ UCIBIO/REQUIMTE, PORTO4AGEING-Competence Center on Active and Healthy Ageing of the University of Porto, Faculty of Pharmacy of the University of Porto, Porto, Portugal and ${ }^{2}$ ICBASAbel Salazar Institute of Biomedical Sciences, University of Porto, Porto, Portugal; ${ }^{2}$ CINTESIS-Center for Research in Health Technologies and Services, Porto, Portugal and Faculty of Medicine of the University of Porto, Porto, Portugal; (luismidao@gmail.com)
\end{abstract}

Aim: In this work, we analysed how COVID-19 affected medication adherence behaviour, after 1 year of pandemic, in Portugal.

Methods: A cross-sectional online survey was conducted between 1st March and 3rd April 2021, which assessed: demographics, psychological status, illness status, medication adherence (MARS-9) and changes during the outbreak, adherence to healthy lifestyles and to COVID-19 preventive measures.

Results/discussion: Of the 1202 participants, 476 who were taking at least one medication prescribed by the doctor were selected. Of these, $78.2 \%$ were female, and the mean age was $40.3 \pm 17.9$ years old. About $74.2 \%$ were classified as being more adherent. Comparing with being single, widows were three times more prone to be less adherent (aOR 3.390 [1.106-10.390], $\mathrm{p}=0.033$ ). Comorbid patients were 1.8 times (aOR 1.824 [1.155-2.881], $\mathrm{p}=0.010$ ) more prone to be less adherent. COVID-19 led to changes in adherence in $14.1 \%$ of the participants $(8.2 \%$ improved, while $5.9 \%$ had worsen adherence results). Finally, participants who reported that COVID-19 negatively impacted their adherence were 5.6 times more prone to be less adherent, comparing with those who reported no changes due to the pandemic (aOR 5.576 [2.420-12.847], p < 0.001). None of the other variables showed to be significantly associated with MARS-9 classification.

Conclusion: This study shows that $14.1 \%$ of participants changed medication adherence behaviours during COVID-19. This highlights the need of continuous measurements of adherence, as it is a dynamic process and being careful using this data on future research.
Potentially inappropriate prescribing and related costs: results from an educational intervention program in Italy

Sara Mucherino ${ }^{1}$, Manuela Casula ${ }^{2,3}$, Federica Galimberti ${ }^{2,3}$, Ilaria Guarino ${ }^{1}$, Elena Olmastroni ${ }^{2}$, Valentina Orlando ${ }^{1}$, Elena Tragni ${ }^{2}$, Enrica Menditto ${ }^{1}$, on behalf of the EDU.RE.DRUG Group

${ }^{1}$ Center of Drug Utilization and Pharmacoeconomics (CIRFF), Department of Pharmacy, Federico II University of Naples, Naples, Italy, ${ }^{2}$ Epidemiology and Preventive Pharmacology Service (SEFAP), Department of Pharmacological and Biomolecular Sciences, University of Milan, Milan, Italy, ${ }^{3}$ IRCCS MultiMedica, Via Milanese 300, 20,099, Sesto S. Giovanni (MI), Italy (sara.mucherino@unina.it)

Aim: To assess impact on costs variation of educational interventions aimed to improve the appropriate drug use.

Methods: Educational interventions were directed to general practitioners (GPs) and their patients of four local health units (LHUs) of Campania (South) and four of Lombardy (North), in the framework of the EDU.RE.DRUG Project. GPs and patients were assigned to an intervention-arm: intervention on GPs and patients; intervention on GPs; intervention on patients; no intervention (control-group). Analyses were performed among elderly population ( $\geq 65$ years), received at least one inappropriate prescription listed in the EDU.RE.DRUG (ERD)-List and focused on ten most frequently potentially inappropriate prescriptions (PIPs). Differences in prevalence between the pre- and post-intervention period were assessed for each LHU. Cost analysis was performed using a bottom-up approach. Costs were related to the reference period (pre- or post-intervention).

Results: Among all intervention-arms glibenclamide and ticlopidine prescriptions significantly decreased in the post-intervention period for both North and South. Post-intervention on GPs and patients, avoidable cost ERD-related was 1.5 billion $€$ in the South versus $515,000 €$ in the North; post-intervention on GPs, avoidable costs were similar for North and South (1.9 billion€); post-intervention on patients, avoidable cost was $715,000 €$ in the versus $678,000 €$ in the North. Higher costs were recorded for proton pump inhibitors in both macro-regions.

Conclusion: Findings identify a high prevalence of PIPs in Italy, with significant cost consequences as unmet need for educational interventions aimed at improving prescribing practice and avoiding economic waste.

\section{Measurement properties of existing patient reported outcome measures on medication adherence: a systematic review}

\section{Dan V. Blalock ${ }^{1,2}$, Livia Ooi ${ }^{3}$, Dionne Hui Fang Loh $^{4}$, Lian Leng Low ${ }^{5}$, Hayden Barry Bosworth ${ }^{1,2,6,7}$, Julian Thumboo ${ }^{8,9,10}$, Yu Heng Kwan ${ }^{8}$}

${ }^{1}$ Center of Innovation to Accelerate Discovery and Practice Transformation (ADAPT), Durham Veterans Affairs Health Care System, Durham, NC, USA; ${ }^{2}$ Department of Psychiatry and Behavioral Sciences, Duke University School of Medicine, Durham, NC, USA; ${ }^{3}$ Department of Pharmacy, National University of Singapore, Singapore; ${ }^{4}$ PULSES Centre Grant, SingHealth Regional Health System; ${ }^{5}$ Department of Family Medicine and Continuing Care, Singapore General Hospital, Singapore; ${ }^{6}$ Department of Population Health Sciences, Duke University Medical Center, 2200 W. Main St., Durham, 
NC 27,705, USA; ${ }^{7}$ School of Nursing, Duke University Medical Center, 307 Kent Dr., Durham, NC 27,710, USA; ${ }^{8}$ Program in Health Services and Systems Research, Duke-NUS Medical School Singapore; ${ }^{9}$ Department of Rheumatology and Immunology, Singapore General Hospital, Singapore; ${ }^{10}$ NUS Yong Loo Lin School of Medicine, National University of Singapore, Singapore (daniel.blalock@duke.edu)

Aims: Many patient-reported outcome measures (PROMs) have been developed to measure medication adherence, specifically the implementation phase-defined as the extent to which a patient's actual dosing corresponds to the prescribed dosing regimen. This study evaluates the quality of the PROMs used to measure medication adherence.

Methods: Preferred Reporting Items for Systematic Review and Meta-analysis (PRISMA) guidelines were used. Relevant articles were retrieved from Embase ${ }^{\circledR}$, PubMed ${ }^{\circledR}$, Cochrane Library, Web of Science, and CINAHL databases. PROMs were evaluated with the COnsensus-based Standards for the selection of health Measurement Instruments (COSMIN) guidelines.

Results: 121 Unique medication adherence PROMs from 214 studies were identified. "Hypotheses testing for construct validity" and "internal consistency" were the most frequently described measurement properties. PROMs with at least "moderate" level of evidence for $\geq 5$ measurement properties included: Adherence Starts with Knowledge 20 (ASK-20), Compliance Questionnaire-Rheumatology (CQR), General medication adherence scale (GMAS), Hill-Bone Scale, Immunosuppressant therapy barrier scale (ITBS), Medication Adherence Reasons Scale (MAR-Scale) revised, 5-Item Medication Adherence Rating Scale (MARS-5), 9-Item MARS (MARS-9), 4-item Morisky Medication Adherence Scale (MMAS-4), 8-item MMAS (MMAS-8), Self-efficacy for Appropriate Medication Adherence Scale (SEAMS), Satisfaction with iron chelation therapy (SICT), test of the adherence to inhaler (TAI), and the Voils questionnaire. Measures focused primarily on the implementation phase of adherence.

Discussion: This study identified 121 PROMs for medication adherence, and provided synthesized evidence for the measurement properties for these PROMs.

Conclusion: The findings from this study may assist clinicians and researchers in selecting suitable PROMs to assess medical adherence, specifically the implementation phase.

\section{Addressing differential medication non-adherence in pragmatic trials with point-treatment settings: a simulation study}

\author{
Hossain M. B. ${ }^{1}$, Karim M. E. ${ }^{1}$ \\ ${ }^{1}$ University of British Columbia, Canada (belal.hossain@ubc.ca)
}

Introduction: Differential but one-sided non-adherence is common in many pragmatic trials. The inverse probability-weighted per-protocol (IPW-PP) method can address this issue but assumes no unmeasured confounding. If some necessary confounders are unmeasured, instrumental variable (IV)-based methods [two-stage least square (2SLS), two-stage residual inclusion (2SRI), nonparametric causal bound (NPCB)] can be used, but they require exclusion restriction assumption. Although all these four methods aim to address the same problem, comprehensive simulations to compare their performance are absent in the literature.

Aim: To compare the performance of IPW-PP, 2SLS, 2SRI, NPCB methods when complete adherence in the control group vs. differential non-adherence in the treatment group.
Methods: We performed extensive simulations when (Setting-1) confounding is present, (Setting-2) confounder is unmeasured but exclusion restriction met, and (Setting-3) exclusion restriction violated. We compared the performance in terms of bias, standard error, and $95 \%$ confidence interval coverage probability.

Results: For Setting-1, IPW-PP produces small bias and nominal coverage, but 2 SLS/2SRI have high bias beyond $60 \%$ non-adherence. For Setting-2, IPW-PP always produces biased estimates, but 2SLS/ 2SRI produce bias estimates only beyond $60 \%$ non-adherence. For Setting-3, 2SLS/2SRI perform the worst. NPCB has wider bounds in all settings. We also analyze a two-arm trial estimating vitamin-A supplementation effect on childhood mortality after addressing differential non-adherence.

Conclusion: We need to be cautious using the IPW-PP when there is strong unmeasured confounding and avoid using 2SLS/2SRI methods when exclusion restriction assumption is violated or high-differential non-adherence rate.

\section{Frequency of changes in medication intake behavior}

\author{
Pironet A. ${ }^{1}$, Tousset E. ${ }^{1}$, Vrijens B. ${ }^{1}$ \\ ${ }^{1}$ AARDEX Group, Belgium (antoine.pironet@aardexgroup.com)
}

Aim: A patient's medication intake behavior often changes over time, improving or worsening. Electronic monitoring of medication adherence provides a timestamp corresponding to each medication intake. This work aimed at making use of this information at intake level to detect changes in medication intake behavior and quantify the frequency of changes.

Methods: Electronic monitoring data for all subjects under a oncedaily dosing regimen and monitored for at least 180 days were extracted from the MEMS ${ }$ Adherence Knowledge Centre. A data science-based proprietary algorithm was used to analyze the electronic monitoring data of each subject and indicated when behavior changes occurred.

Results: Data from 7595 subjects were retrieved. Of these, 5012 $(66 \%)$ experienced at least one behavior change during the monitoring. The median frequency of behavior change is two times a year (minimum: 0, maximum: 26). The median time to first behavior change is 124 days.

Discussion: These results show that most subjects change their medication intake behavior during a trial. Consequently, it is important to monitor these behaviors. Using such a system, the subject's healthcare provider can be informed when a behavior change is detected. In addition, when analyzing adherence data, medication adherence metrics computed on homogeneous intake behavior periods make much more sense than those computed on the whole, inhomogeneous monitoring period.

Conclusion: An algorithm for detection of changes in medication intake behavior was applied to electronic monitoring data and showed that most subjects change their behavior during a trial.

\section{Association between reported barriers to medication adherence and electronically monitored adherence in kidney transplant recipients}

\author{
Carusone, LiAnna ${ }^{1}$; Dahhou, Mourad ${ }^{1}$; Zhang, Xun $^{1}$; \\ Foster, Bethany J. ${ }^{1}$ \\ ${ }^{1}$ McGill University, Canada (lianna.carusone@mail.mcgill.ca)
}


Aim: To determine the association between perceived barriers to adherence, as measured using the adolescent medication adherence survey (AMBS), and the implementation phase of immunosuppressive medication adherence.

Methods: This is a secondary analysis of data from the pre-intervention 3-month run-in of the TAKE-IT trial. Adherence was monitored electronically among 136 kidney transplant recipients 11-24 years and scored $0 \%, 50 \%$, or $100 \%$ on each day of observation based on the proportion of prescribed doses taken. Standard deviation of tacrolimus trough levels for the interval 3 months before baseline to end of run-in was assessed as a secondary outcome (adherent if $\mathrm{SD}<2$ ). Multinomial logistic regression with GEE was used to estimate the association between AMBS total score and adherence. Models were adjusted for age, race, gender, and household income. Additional models considered the association between adherence and barrier load ( $<3$ vs. ${ }^{3} 3$ barriers), total number of barriers and AMBS sub-scale scores.

Results: Odds of adherence were not associated with AMBS total score $(\mathrm{OR}=1.00$ [95\% CI $0.98-1.02])$, total number of barriers $(\mathrm{OR}=1.01[95 \%$ CI $0.92-1.10])$, barrier load $(\mathrm{OR}=1.24$ [95\% CI $0.78-1.99]$ ) or any AMBS sub-scale score. There were no associations between tacrolimus SD-based adherence and any of the AMBS measures.

Discussion: A high AMBS disease frustration/adolescent issues subscale score was previously associated with poorer adherence (assessed by self-reported adherence and drug level variability).

Conclusion: No associations between AMBS total or subscale scores and medication adherence were seen in this independent data set.

\section{Emerging bioanalytical methods for drug monitoring in patients receiving chronic drug therapies: a scoping review}

Zijp T. R. ${ }^{1}$, Izzah Z. ${ }^{1,2,4}$, Åberg C. ${ }^{4}$, Gan C. T. ${ }^{5}$, Bakker S. J. L. ${ }^{6}$, Touw D. J. ${ }^{1,3,4}$, van Boven J. F. M. ${ }^{1,3}$

${ }^{1}$ University of Groningen, University Medical Center Groningen, Department of Clinical Pharmacy \& Pharmacology, Groningen, The Netherlands, ${ }^{2}$ Universitas Airlangga, Faculty of Pharmacy, Department of Pharmacy Practice, Surabaya, Indonesia, ${ }^{3}$ Medication Adherence Expertise Center of the Northern Netherlands (MAECON), Groningen, The Netherlands, ${ }^{4}$ University of Groningen, Groningen Institute of Pharmacy, Department of Pharmaceutical Analysis, Groningen, The Netherlands, ${ }^{5}$ University Medical Center Groningen, Respiratory Diseases and Lung Transplantation, Groningen, The Netherlands, ${ }^{6}$ University Medical Center Groningen, Department of Internal Medicine, Division of Nephrology, Groningen, The Netherlands (t.r.zijp@umcg.nl)

Introduction: Direct quantification of drug concentrations in the body allows medication adherence measurement but also therapeutic drug monitoring (TDM). Historically this required patient unfriendly blood drawings, but in recent years multiple less-invasive methods were developed: dried blood spots (DBS), saliva and hair analyses.

Aim: This review aims to provide an overview of novel drug quantification methods for TDM and medication adherence monitoring (MAM), focusing on clinical validation for patients using chronic drug therapies.

Methods: A scoping review was performed using a systematic search in three electronic databases covering 2000-2020. Screening and inclusion were performed by two independent reviewers in Rayyan. Data from the articles were extracted in a RedCap database. Main outcome was clinical validity, based on study sample size, means of cross-validation and authors' method description.
Results: A total of 4590 studies were identified and 175 articles were finally included; 57 on DBS, 66 on saliva and 55 on hair analyses. Most reports were within Neurological diseases (36.6\%), Infectious diseases $(30.8 \%)$, and Transplantation (13.7\%). An overview was generated of all measured drugs with clinical validation status and whether these methods were used for TDM or MAM.

Conclusion: TDM and MAM are increasingly possible without traditional invasive blood sampling: the strengths and limitations of DBS, saliva and hair differ and all have shown potential for drug monitoring. To ensure strength of future evidence, we recommend standardization of the clinical method validation.

\section{Constructing effective text messages to promote adherence in chronically-ill individuals: a theoretical and practical exploration}

Gessl, Alessandra S. ${ }^{1}$; Nakata, Cheryl C. ${ }^{2}$; Xiao, Yazhen $^{3}$; Cui, Anna Shaojie ${ }^{4}$; Crawford, Stephanie Y. ${ }^{5}$; Sharp, Lisa K. ${ }^{5}$; Spanjol, Jelena ${ }^{1}$

${ }^{1}$ Munich School of Management, Ludwig-Maximilians-Universität München, Germany; ${ }^{2}$ Bryan School of Business and Economics, University of North Carolina at Greensboro, United States; ${ }^{3}$ Haslam College of Business, University of Tennessee at Knoxville, United States; ${ }^{4}$ Department of Marketing, University of Illinois at Chicago, United States; ${ }^{5}$ College of Pharmacy, University of Illinois at Chicago, United States (gessl@bwl.lmu.de)

Introduction: Mobile phone text messages are often employed towards promoting medication adherence. The construction (wording, content, and structure) of text messages remains underexplored, despite evidence from psychology and consumer behavior studies indicating effects on behavioral responses to adherence-promoting text messages. According to construal theory, greater temporal distance to a targeted behavior is related to higher desirability of the behavior.

Aim: The aim of this study is to explore how temporal framing of adherence-promoting messages influences perceived text message effectiveness, desirability and feasibility of adherence, and adherence intentions in chronically-ill individuals.

Methods: We recruited adults $(\mathrm{n}=2881)$ with hypertension from the United States and administered an online text-message intervention and survey. Participants were randomly allocated to one of four temporal frames (today, tomorrow, next month, future) from temporally near (today) to temporally distant (future), and were presented with a text message stimulus. Perceived text message effectiveness, desirability and feasibility of adherence, and adherence intentions were measured via multi-item scales.

Results: Temporal distance of the text message made a difference in perceived message effectiveness and other outcome measures. The two temporal extremes (today and future) were perceived as more effective than the mid-range temporal frames (tomorrow and next month).

Discussion: Text message interventions should use temporal extremes rather than mid-range frames to encourage medication adherence.

Conclusion: Further research should investigate whether the effect of temporal frames is dependent on message recipient characteristics. 


\section{Refining the SPUR tool: interim analysis of UK and French studies}

\author{
Kevin Dolgin ${ }^{1}$, Joshua Welss ${ }^{2}$ \\ ${ }^{1}$ Observia, General Manager of Observia, Paris, France); ${ }^{2}$ School of \\ Life Sciences, Pharmacy and Chemistry, Kingston University) \\ (kevin.dolgin@observia-group.com)
}

Aim: To combine the interim results of two separate studies to assess
the validity of the tool and the methods used to assess it.
Methods: We administered the SPUR and the medication adherence
rating scale (MARS) questionnaires to 600 T2DM patients in the UK
and France. Pearson correlation coefficients were then examined
between the questionnaires and for each with respect to 6-month
MPR. The analysis was done on the first 178 UK patients and the first
242 French patients.
Results: In both countries, SPUR was highly correlated with MARS
(p $<0.01$ in both cases). In the UK, both MARS and SPUR were
correlated with MPR (p $<0.01$ in both cases). In France, neither
MARS nor SPUR was significantly correlated to MPR. However,
when the 88 patients with MPR of 100 were removed, SPUR showed
significant correlation to the remaining patients (PCC = 0.17,
p = 0.01 ), whereas MARS remained uncorrelated. Restricting analy-
sis only to patients with MPR $<100$ among the UK patients did not
significantly change the correlations of either MARS or SPUR.
Discussion: These results further validate the ability of SPUR to
assess elements of the risk of non-adherence. The differences in the
relationships of the various measures to MPR in the UK and in France
may indicate that researchers should consider structural and cultural
differences when using short-term MPR.
Conclusion: SPUR represents a viable new tool to measure Conclusion:
adherence.

\section{Development of a checklist to assess adherence barriers to $\mathrm{HCV}$ treatment in ambulatory setting}

\section{Barbati Selina $^{1}$, Strasser Hannes ${ }^{2}$, Hersberger Kurt ${ }^{1}$, Arnet Isabelle ${ }^{1}$}

${ }^{1}$ Department of Pharmaceutical Sciences, Pharmaceutical Care Research Group, University of Basel, Petersplatz 15, P.O. Box 2148, CH-4001 Basel, Switzerland; ${ }^{2}$ University Psychiatric Clinics Basel, Wilhelm Kleinstrasse 27, CH-4012 Basel, Switzerland (selina.barbati@unibas.ch)

Introduction: The WHO has set a goal to eradicate hepatitis $\mathrm{C}$ virus (HCV) by the year 2030. Barriers to HCV care are still present at the level of patients, providers, and systems. Identifying potential barriers before treatment initiation is crucial to ensure optimal adherence to $\mathrm{HCV}$ medicines. The economic impact of non-adherence to $\mathrm{HCV}$ is increased given the high costs of treatment.

Aim: To develop a checklist assessing adherence barriers in ambulatory patients who initiate HCV treatment.

Methods: Barriers to HCV treatment mentioned by the key risk group of people who inject drugs were searched in literature. From 34 retrieved articles, we extracted 80 modifiable barriers and developed descriptive themes. The completeness of thematic domains was checked with a framework on patients' lived experience with medicines (PLEM). The three prime questions from the Indian Health Service were added as validated conversation openers.

Results: Our preliminary checklist consists of 30 items within 4 categories (personal difficulties and barriers to treatment, personal motivation to be treated, knowledge about the disease, received information about the medicine) at patient, provider, and system level.

Discussion: We generated items with a triple approach from literature, a framework on patients' lived experience with medicines, and counseling questions. By doing so, we obtained a complete checklist for pharmacy practice.

Conclusion: Refinement of our checklist will be defined with $\mathrm{HCV}$ patients in future steps. Targeted interventions matching the barriers should also be defined and implemented in daily practice.

\section{Czech language translation of the ABC Taxonomy for medication adherence}

\author{
Mala-Ladova K. ${ }^{1}$, Voriskova E. ${ }^{1}$, Kostalova B. ${ }^{1}$, Maly J. ${ }^{1}$ \\ ${ }^{1}$ Department of Social and Clinical Pharmacy, Faculty of Pharmacy in \\ Hradec Kralove, Charles University, Czech Republic \\ (ladovaka@faf.cuni.cz)
}

Aim: There is a need for cross-cultural translations of the original English language ABC Taxonomy. The aim was to establish a consensus of medication adherence terminology in the Czech language. Methods: The Czech language literature was reviewed before January 2021 releasing 122 papers published between 1998 and 2020. Based on that, a questionnaire for a three-round online Delphi survey as well as a list of panelists invited in the survey were generated. The survey was held between January and May 2021 and consensus was defined according to the acceptance rate. The questionnaire contained items focused on the translation of seven terms and their definitions related to $\mathrm{ABC}$ Taxonomy.

Results: Out of 106 panelists contacted in the first round, $46(44.4 \%)$ responded. A consensus ( $>85 \%$ ) of two definitions was established. In the second round, 32 respondents established the consensus $(>75 \%)$ of one term and four definitions. The third round was responded by 24 panelists and moderate $(>50 \%)$ to strong $(>95 \%)$ consensus for the rest of terms and definitions was established. Seven terms were as follow: adherence $\mathrm{k}$ léčbě, zahájení léčby, zavedení léčby, přerušení léčby, setrvání na léčbě, management adherence, obory související s adherencí.

Discussion and conclusion: The consensus for translation of all Czech terms and definitions related to medication adherence can facilitate comparisons between studies and dialogue among scientists and clinicians.

Study was supported by Charles University (SVV 260 551).

\section{Urine acetyl-Ser-Asp-Lys-Pro measurement as a universal biomarker to assess adherence to angiotensin converting enzyme inhibitors}

\section{Kably B. ${ }^{1}$, Derobertmasure A. ${ }^{1}$, Billaud E. ${ }^{1}$, Blanchard A. ${ }^{2}$, Boutouyrie P. ${ }^{1}$, Azizi M. ${ }^{3}$}

${ }^{1}$ Hopital Europeen Georges Pompidou - Pharmacology Unit, Paris, France; ${ }^{2}$ Hopital Europeen Georges Pompidou - Centre d'investigations cliniques, Paris, France; ${ }^{3}$ Hopital Europeen Georges Pompidou - Hypertension Unit, Paris, France (ladovaka@faf.cuni.cz)

Chemical adherence testing (CAT) using liquid chromatography/mass spectrometry (LCMS) techniques have emerged as new methods of adherence assessment showing promising results. Their implementation requires the development of large detection panels of drugs. Thus, the use of acetyl-Ser-Asp-Lys-Pro (AcSDKP), a proven 
marker of adherence to angiotensin-converting-enzyme inhibitor (ACEI) treatment, could be useful.

Our aim was to validate urine measurements of AcSDKP against active metabolites of various ACEI measured simultaneously by LCMS.

We first studied the time-dependent relationships between urinary perindoprilat and AcSDKP in healthy subjects. We then compared the sensitivity and specificity of urinary AcSDKP vs. 3 ACEI metabolites taken as the gold standard to detect nonadherence in spot urine samples from a prospective cohort of hypertensive outpatients.

First, we found that the urine profiles of AcSDKP and perindoprilat excretion were similar, and showed a significant log-linear correlation. In the second study, we found a similar correlation with each of the three ACEI metabolites. The sensitivity and specificity of urine AcSDKP were $92.2 \%$ and $100 \%$ for adherence assessment. We observed a difference in the evaluation of good adherence between ACEI metabolites (85.7\%) and AcSDKP (79.0\%).

Urine AcSDKP and ACEI metabolites levels are closely related. For adherence assessment, the discrepancies observed in some samples were mainly due to analytical reasons, AcSDKP attaining undetectable concentrations more rapidly.

Spot urine AcSDKP measurement by LCMS is a reliable marker of ACEI intake and could substitute ACEI metabolites detection.

\section{Communication and information about non-medical medication switches by the pharmacy team: patient's needs and preferences}

\author{
Schackmann L., Heringa M., Koster E., van Dijk L., Vervloet M. \\ ${ }^{1}$ Nivel (The Netherlands), University of Groningen (The Nether- \\ lands), ${ }^{2}$ SIR Institute for Pharmacy Practice and Policy (The \\ Netherlands), ${ }^{3}$ UPPER/Universiteit Utrecht (The Netherlands) \\ (1.schackmann@nivel.nl)
}

Introduction: The pharmacy team often has to deliver a message about a medication switch. In order to have constructive conversations about medication switches, it is important for the information provision to be adapted to the patient's needs. This may lead to better treatment adherence.

Aim: To assess patients' needs and preferences concerning information about non-medical medication brand switches.

Methods: Online questionnaires were distributed via the Dutch patient organization for cardiovascular diseases panel. The questionnaire addressed communication, information provision and needs around conversations regarding medication switches. Descriptive analysis has been performed.

Results: 717 People completed the questionnaire, of whom 532 (75\%) had at least one medication switch in the past year. Most respondents $(90 \%)$ wanted to receive information, whereby written information before medication dispense $(38 \%)$ or verbally during medication dispense (42\%) was preferred. About half $(54 \%)$ indicated that they had received enough information. About 59\% of these respondents indicated that they had been informed by the pharmacy about why the switch took place, $42 \%$ were informed about the (same) effect of the medication. These were also the two most frequently mentioned topics on which patients would like to receive information.

Discussion: Patients with cardiovascular disease were enquired, though medication switches may have other effects on different patient-groups or medication types.

Conclusion: When information is given about a medication switch, in this case about $50 \%$ of the respondents, this is often the information that patients need.

\section{Assessing medication adherence to drugs for obstructive airway diseases (ATC R03): an observational study}

Armando L. G. ${ }^{1}$, Basso L. ${ }^{1}$, Traina S. ${ }^{1}$, Baroetto Parisi R. ${ }^{2}$, Remani E. ${ }^{2}$, Boscaro V. ${ }^{1}$, Miglio G. ${ }^{1,3}$, Cena C. ${ }^{1}$

${ }^{1}$ Dipartimento di Scienza e Tecnologia del Farmaco-Università degli Studi di Torino, Italy; ${ }^{2}$ Struttura Complessa Farmacia Territoriale ASL TO4-Regione Piemonte, Italy; ${ }^{3}$ Centro di Competenza sul Calcolo Scientifico C3S-Università degli Studi di Torino, Italy (lucrezia.armando@edu.unito.it)

Aim: To describe the use of R03-drugs in two different settings: a Casa della Salute (CdS) in the local health unit ASLTO4 (Piedmont, Italy) and the traditional outpatient setting of the ASLTO4.

Methods: Data on drugs dispensed between June 2018 and July 2019 were provided by the ASLTO4. Medication adherence was calculated over 13 months with AdhereR using the continuous medication availability (CMA) and the defined daily doses. CMA values were used to categorize patients as adherent $(\geq 80 \%)$, partially adherent or non-adherent $(<20 \%)$. Prescriptive appropriateness evaluation was also conducted in a subgroup of these patients.

Results: A total of 832 patients were analysed. Individual CMA values showed a wide variability: adherence to R03AL- and R03DAdrugs was significantly higher in the CdS patients, while it was higher for R03AC-drugs in the control group. Most patients in both groups were non-adherent to R03-drugs. Prescriptions to patients diagnosed with asthma or chronic obstructive pulmonary disease $(n=45)$ were analysed: medication was consistent with the latest version of the GINA and GOLD guidelines for 28 patients, while the remaining 17 patients lacked of a long-acting bronchodilator, had more than one long-acting beta-agonists or had $\geq 5$ different R03-drug classes codispensed.

Discussion: Inappropriate therapies and poor adherence to R03-drugs could lead to an increased risk of hospitalizations for respiratory failure.

Conclusion: Our analysis highlights the need for interventions to improve prescriptive appropriateness and reduce non-adherent behaviors.

\section{Financial interventions to improve medication adherence: a narrative review}

\section{Dan V. Blalock ${ }^{1,2}$, Anna Hung ${ }^{1,3}$, Julie Miller ${ }^{1,3}$,} Jaime McDermott ${ }^{4}$, Hannah Wessler ${ }^{3}$, Leah L. Zullig ${ }^{1,3}$

${ }^{1}$ Center of Innovation to Accelerate Discovery and Practice Transformation (ADAPT), Durham Veterans Affairs Health Care System, Durham, NC, USA; ${ }^{2}$ Department of Psychiatry and Behavioral Sciences, Duke University School of Medicine, Durham, NC, USA; ${ }^{3}$ Department of Population Health Sciences, Duke University Medical Center, Durham, NC, USA; ${ }^{4}$ Duke University Hospital, Durham, NC, USA (daniel.blalock@duke.edu)

Introduction: Out-of-pocket costs are consistently reported as one of the largest barriers to medication adherence.

Aims: To review the qualities of financial interventions aimed at improving medication adherence.

Method: Relevant MeSH terms were searched in PubMed ${ }^{\circledR}$ and Web of Science ${ }^{\circledR}$, yielding 395 articles. Abstracts were reviewed by two reviewers, leading to 26 articles for full text review. 15 Met inclusion criteria. 
Results: 13 Studies were from the US; 3 were prospective. Sample sizes ranged from 38 to 1.1 million. Populations included patients with cardiovascular chronic conditions $(n=5)$, other chronic conditions $(n=3)$, cancers $(n=3)$, post-hospital procedures $(n=3)$, and depression $(n=1)$. Financial interventions included: provision of a copayment voucher $(n=8)$, connection with a medication assistance program $(n=7)$, connection to specialty pharmacy services for financial resources and support $(n=4)$, and/or direct provision of free medications $(n=2)$. Most interventions measured the implementation phase of medication adherence $(n=12)$. Some measured non-persistence $(n=2)$, initiation $(n=1)$, or were indiscernible with regard to phase $(n=4)$. Thirteen studies used some form of claims or pharmacy-based adherence measure, $\mathrm{n}=1$ study relied on self-report and blister pack, and $\mathrm{n}=1 \mathrm{did}$ not describe the method of adherence. Twelve studies concluded that the measured financial intervention positively impacted medication adherence.

Discussion: Despite large sources of heterogeneity, financial interventions may be beneficial for improving medication adherence.

Conclusion: Financial interventions exist in a variety of formats for different disease populations, but overall may be beneficial for improving medication adherence.

\section{Adherence to cardiovascular disease risk factor medications among patients with cancer: a systematic review}

\section{Zullig Leah L. ${ }^{1,2,3}$, Shahsahebi Mohammad ${ }^{3}$, Avecilla Renee ${ }^{3}$,} Whitney Colette $^{3}$, Mills Coleman ${ }^{3}$, Oeffinger Kevin C. ${ }^{3}$

${ }^{1}$ Center of Innovation to Accelerate Discovery and Practice Transformation, Durham Veterans Affairs Health Care System, USA, ${ }^{2}$ Department of Population Health Sciences, Duke University School of Medicine, USA, and ${ }^{3}$ Center for Onco-Primary Care, Duke Cancer Institute, USA (Leah.zullig@duke.edu)

Introduction: The most common cause of mortality for many cancer survivors is cardiovascular disease (CVD).

Aim: To provide an evidence-based understanding of adherence to medications for the management of CVD risk factors among cancer survivors.

Methods: We systematically searched for articles indexed in MEDLINE, Embase, Cochrane, PsycINFO, Scopus for articles published from inception to October 31, 2019. English language, original research studies that assessed medication adherence to common CVD risk factors among cancer survivors. Two reviewers extracted study data and assessed risk of bias using the mixed methods appraisal tool. Results: Of the 20 studies included, 55\% focused on multiple cancer types and $70 \%$ used pharmacy-based adherence measures. Only two were prospective studies. Adherence was variable across cancer types and specific CVD risk factors. Among the studies that examined changes in comorbid medication adherence, most noted a decline in adherence following a diagnosis of cancer and over the course of the cancer experience (during and following active treatment). There was a high focus on breast cancer populations, which may not be generalizable to all cancer types. Studies did not differentiate between phases of adherence.

Discussion: Medication adherence to CVD risk factor-related medications is low among cancer survivors and declines over time. Current literature in this area largely does not reference the ABC Taxonomy. Conclusions: Given the risk for CVD-mortality among cancer survivors, testing of interventions aimed at improving adherence to noncancer medications is critically needed.
Improving adherence to cardiovascular and diabetes treatments in primary care: pilot of a complex intervention

Corral-Partearroyo C. ${ }^{1}$, Sanchez-Viñas A. ${ }^{2}$, Gil-Girbau $\mathbf{M}^{3}$, Peñarrubia-María M. T. ${ }^{4}$, Aznar-Lou I. ${ }^{2}$, Gallardo-González M. C. ${ }^{4}$, Rubio-Valera M. ${ }^{2}$, Parc Sanitari ${ }^{2}$

${ }^{1}$ Sant Joan de Déu Research Institute, Spain; ${ }^{2}$ Sant Joan de Déu Research Institute, Consortium for Biomedical Research in Epidemiology \& Public Health (CIBERESP), Spain; ${ }^{3}$ Sant Joan de Déu Research Institute, Primary Care Prevention and Health Promotion Research Network (redIAPP), Spain; ${ }^{4}$ Catalan Institute of Health, Primary Care Research Institute (IDIAP Jordi Gol), Spain (c.corral@pssjd.org)

Aim: (i) To evaluate the feasibility and acceptability of the IMA intervention, a multidisciplinary and shared decision-making intervention to improve adherence and clinical outcomes, and (ii) to explore the availability and quality of registries for initiation and secondary outcomes (implementation, persistence, clinical data and costs).

Methods: A pilot study was conducted over twelve weeks in five primary care centres in Catalonia (Spain), with an embedded process evaluation. We conducted 29 semi-structured interviews with professionals (physicians, nurses and pharmacists) and patients. Realworld data from electronic records will be used to test the intervention outcomes.

Results: The intervention components and implementation strategies were feasible and acceptable for participants. However, according to their feedback, modifications on some of the components of the intervention, such as training of professionals and decision aids for patients, should be considered before launching a cluster-RCT. Information on the availability and quality of data on outcomes will be presented at the conference.

Discussion: Involving patients in the decision-making process is key to achieving better clinical outcomes. However, patient-centred care requires modifications of the clinical practice. The process evaluation has allowed us to identify the barriers and facilitators to implement the intervention and adapt to a context affected by the COVID-19 pandemic.

Conclusions: The IMA intervention is feasible and had good acceptability among professionals and patients. This pilot study has identified opportunities for improving its design before the clusterRCT to evaluate its effectiveness and cost-effectiveness.

\section{No hesitancy to vaccination schedule in HIV + Italian people: a local survey}

\author{
Guastavigna M. ${ }^{1}$, Farenga M. ${ }^{1}$, Orofino G. C. ${ }^{1}$ \\ ${ }^{1}$ Amedeo di Savoia Hospital, Infectious Diseases Division A, Turin, \\ Italy (martaguastavigna@gmail.com)
}

Introduction/aim: HIV + -patients present frequent and serious infectious diseases, thus vaccinations are very important and effective. An implementation of vaccination policy started in our center. Aim of this survey is to explore vaccination hesitancy (confidence, complacency, convenience).

Methods: During follow-up at the Infectious Diseases Clinic in Turin, 855 patients were prescribed appropriate vaccines schedule (anti-pneumococcus, anti-meningococcus $\mathrm{B}$ and $\mathrm{C}$, anti-hepatitis $\mathrm{A}$ and $\mathrm{B}$, anti-Haemophilus influenzae, anti-HPV). They were referred to 
local vaccination centers. Some vaccines could be performed at our center to promote adherence in patients hesitant for convenience.

Results: 643 (74\%) Male patients, mean age 51 years and $574(89 \%)$ Italian; 189 (26\%) female patients, mean age 50 years and $133(70 \%)$ Italian. All subjects were receiving antiretroviral therapy. Male group: $525(82 \%)$ underwent at least one vaccination, $108(17 \%)$ didn't undergo any vaccination, $10(1 \%)$ refused vaccinations. Female group: $141(75 \%)$ underwent at least one vaccination, 36 (19\%) didn't undergo any vaccination, $12(6 \%)$ refused vaccinations.

Discussion: Results show high adherence to vaccination prescription, at least in the initiation phase. More time is required to explore persistence and discontinuation due to length of vaccination schedule and to SARS-CoV2 pandemic. Having created a vaccination habit can have an important educational value for SARS-CoV2 vaccination campaign.

Conclusions: During SARS-CoV2 pandemic (and vaccination against COVID 19), it's important to improve attitudes towards vaccines in HIV + people, since vaccine hesitancy, coverage and efficacy aren't established in this population.

\section{Interventions to reduce healthcare costs of potentially inappropriate prescribing: a systematic review of literature}

\author{
Sara Mucherino ${ }^{1}$, Manuela Casula ${ }^{2,3}$, Federica Galimberti $^{2,3}$, \\ Ilaria Guarino ${ }^{1}$, Elena Olmastroni ${ }^{2}$, Elena Tragni ${ }^{2}$, \\ Enrica Menditto $^{1}$, Valentina Orlando ${ }^{1}$, \\ on behalf of the EDU.RE.DRUG Group.
}

${ }^{1}$ Center of Drug Utilization and Pharmacoeconomics (CIRFF), Department of Pharmacy, Federico II University of Naples, Naples, Italy; ${ }^{2}$ Epidemiology and Preventive Pharmacology Service (SEFAP), Department of Pharmacological and Biomolecular Sciences, University of Milan, Milan, Italy; ${ }^{3}$ IRCCS MultiMedica, Via Milanese 300, 20,099, Sesto S. Giovanni (MI), Italy (sara.mucherino@unina.it)

Introduction: Potentially inappropriate medications (PIM) are associated with increased risk of adverse drug reactions, recognized as determinant of adherence and increased healthcare costs.

Aim: To investigate the state of the art for improving potentially inappropriate prescribing (PIP) and the impact on avoidable costs to healthcare systems.

Methods: A systematic review of literature was conducted according to the PRISMA statement guidelines. PubMed and Embase were queried without time limits, to February 2021. According to the PICO Model, search strategy combined headings and keywords (inappropriate prescribing medications; elderly population; costs evaluation; intervention on health professionals or patients) using AND/OR as Boolean operators. Titles, abstracts and full-texts were screened for their eligibility according to inclusion/exclusion criteria.

Results: Search strategy produced 274 potentially relevant publications. 18 Articles met inclusion criteria and were included in the review. Included studies were divided according to their study design: observational studies, assessing the frequency of PIP and associated avoidable costs $(\mathrm{N}=10)$; trials, containing a specific intervention and observation of outcomes in terms of post-intervention effectiveness and avoided costs $(\mathrm{N}=8)$. PIP prevalence ranged from 21 to $79 \%$. All interventions aimed to improve PIP and related avoidable costs were targeted to physicians. After most interventions, outcomes consisted in more efficient prescribing trend and PIP reduction; however, these interventions were not cost-effective.
Conclusion: This review revealed a high prevalence of prescribing inappropriateness at international level showing that higher avoidable costs were related to higher PIP prevalence.

\section{How concern regarding COVID-19 affected general adherence behaviour}

\begin{abstract}
Almada M. ${ }^{1}$, Midão L. ${ }^{1,2}$, Costa E. ${ }^{1}$, Sampaio R. ${ }^{3}$
${ }^{1}$ UCIBIO/REQUIMTE, PORTO4AGEING-Competence Center on Active and Healthy Ageing of the University of Porto, Faculty of Pharmacy of the University of Porto, Porto, Portugal; ${ }^{2}$ ICBAS-Abel Salazar Institute of Biomedical Sciences, University of Porto, Porto, Portugal; ${ }^{3}$ CINTESIS-Center for Research in Health Technologies and Services, Porto, Portugal and Faculty of Medicine of the University of Porto, Porto, Portugal (luismidao@gmail.com)
\end{abstract}

Aim: SARS-CoV-2 pandemic imposed the adoption of new self-care behaviours. Herein, we analysed how the concern about being infected with COVID-19 affected general adherence behaviour, after one year of pandemic, in Portugal.

Methods: A cross-sectional online survey was conducted between 1st March and 3rd April 2021. The survey instrument assessed: illness status and perception; medication adherence persistence (MARS-9), healthy lifestyles and preventive measures regarding COVID-19 spread.

Descriptive statistics were performed, and univariable and multivariable binary logistic regression models.

Results/discussion: Of 1202 participants, $77.8 \%$ were female, and the mean age was $33.0 \pm 15.8$ years old. Of those who were not infected (88.9\%), $72.0 \%$ reported being concerned while $16.9 \%$ were not concerned about being infected. Compared with being single, widows were 5 times less prone to be concerned with COVID-19 infection (aOR 0.218 [0.091-0.472], $\mathrm{p}=0.007$ ). Participants who knew someone infected were 2 times more concerned (aOR 2.120 [1.150-3.909], $p=0.016$ ) and who felt alone, anxious, or nervous were almost 2 times more concerned about being infected (aOR 1.849 [1.299-2.632], $\mathrm{p}<0.001)$. Finally, individuals who were more concerned were those who followed preventive measures as use of hand sanitiser, objects disinfection and avoided crowded places. We found no statistical differences between being concerned about COVID-19 infection and medication adherence or adoption healthier lifestyles. Conclusion: This study reveals that the concern regarding COVID-19 infection promoted the adoption of preventive measures of COVID19 spread, regardless of changes in adherence to medication and healthy habits.

\section{How to clean adherence electronic monitoring data using a semi-automated procedure?}

\section{Carole Bandiera ${ }^{1}$, Jérôme Pasquier ${ }^{1}$, Isabella Locatelli ${ }^{1}$,} Marie P. Schneider ${ }^{1}$

${ }^{1}$ Suisse (carole.bandiera@unisante.ch)

Cancer patients may take oral oncology treatments. Because of side effects, regimens are often altered either by the oncologist or by the patient. Such deviations must be characterized to establish the real exposure to the treatment.

We aimed at developing a computer script for cleaning electronic monitoring (EM) adherence data based on a set of patients with different types of solid cancers under protein kinase inhibitors (PKI). 
We collected the following raw variables: adherence monitoring start and stop dates; prescribed regimen; expected number of daily monitor openings; observed date and time of each monitor opening; reporting of monitor use deviations (date and reasons).

Based on this information, we developed a R-script to format the data longitudinally (LongiData) within the observation window: date, observed and expected numbers of monitor activations. Deviations are codified (e.g. hospitalization).

We calculated and compared PKI implementation during a 12 -month monitored period using raw and cleaned data.

Over 58 monitors used by 23 males and 17 females, mean age 60 , monitored over a median of 7 months (Q1 3.9; Q3 12.2), implementation was $69 \%$ and $92 \%$ (abs 23\%) with raw and cleaned data, respectively.

Data cleaning allows researchers to avoid data misinterpretation and provides a cleaned dataset for implementation and persistence analyses. The development of a semi-automated procedure saves time, decreases data handling errors, increases reproducibility and documentation.

Such open-source R script can be used in any medication adherence study using EM.

\section{Appropriate drug use indicators: adherence and persistence to chronic therapies in the EDU.RE.DRUG Project}

Olmastroni E. ${ }^{1}$, Galimberti F. ${ }^{1,2}$, Casula M. ${ }^{1,2}$, Catapano A. L. ${ }^{1,2}$, Menditto E. ${ }^{3}$, Tragni E. ${ }^{1}$, on behalf of the EDU.RE.DRUG Group.

${ }^{1}$ Epidemiology and Preventive Pharmacology Service (SEFAP), Department of Pharmacological and Biomolecular Sciences, University of Milan, Milan, Italy; ${ }^{2}$ IRCCS MultiMedica, Via Milanese 300, 20,099, Sesto S. Giovanni (MI), Italy; ${ }^{3}$ Center of Drug Utilization and Pharmacoeconomics (CIRFF), Department of Pharmacy, Federico II University of Naples, Naples, Italy; on behalf of the EDU.RE.DRUG Group (elena.olmastroni@unimi.it)

Introduction and aim: Adherence to treatment is essential for the management of chronic conditions. We aimed at evaluating adherence among incident users of statins, antidiabetic, antihypertensive, and anti-osteoporotic drugs, followed for 6 months.

Methods: Data on patients aged $\geq 40$ years from eight local health units of Lombardy and Campania were retrieved within the EDU.RE.DRUG trial. Two phases of adherence process were assessed: implementation, calculated as PDC (proportion of days covered) for each non-occasional patient; persistence to the chronic treatments.

Results: Occasional statin patients in Lombardy were $27-28 \%$, while in Campania on average 1 patient out of 3 interrupted the therapy after the first prescription. The percentage of adherent $(\mathrm{PDC} \geq 0.80$ ) statin users varied between 48.1 and $56.9 \%$; in Campania, percentages were between 34.2 and $47.2 \%$. Persistent patients were $77.1-81.8 \%$, and $65.2-75.7 \%$, respectively. Patients adhering to antihypertensive drugs were $35.6-49.0 \%$ in Lombardy, $32.4-43.6 \%$ in Campania, while persistence prevalence ranged between 59.5 and $71.1 \%$ and between 58.4 and $68.2 \%$, respectively. For antidiabetic drugs, a similar pattern was observed, with even lower adherence rates observed in Campania. In the two regions, occasional patients represented about a quarter and a third of the new antidiabetic users. About anti-osteoporotic drugs, we observed 38.1-61.3\% of adherent patients in Lombardy and $32.5-47.1 \%$ in Campania, while percentages of persistent patients were $60.8-82.8 \%$ and $61.2-68.4 \%$, respectively.

Conclusion: These low rates of adherence to chronic therapies warrant a multidisciplinary approach, which would require coordinated action between health professionals, researchers, policy makers and patients.

\section{Translation of the ABC Taxonomy on medication adherence into Spanish}

Malo S. ${ }^{1,2}$, Aguilar I. ${ }^{1}$, Rabanaque M. J. ${ }^{1}$, Benrimoj S. I. ${ }^{3}$, Gastelurrutia M. A. ${ }^{3}$, Martinez-Martinez F. ${ }^{3}$, Garcia-Cardenas V.

${ }^{1}$ University of Zaragoza, Spain; ${ }^{2}$ University of Technology Sydney, Australia; ${ }^{3}$ University of Granada, Spain (victoria.garciacardenas @uts.edu.au)

Introduction: A wide range of terms and definitions are used in the literature to refer to medication adherence, leading to confusion and hindering the comparison across studies. This is a common problem in many languages, including Spanish.

Aim: To describe the process and preliminary results obtained during the translation of the ABC Taxonomy for medication adherence into Spanish.

Methods: The process was undertaken following the methods adopted by ESPACOMP. A literature search was conducted in PubMed to identify Spanish synonyms of the ABC Taxonomy. Definitions were forward translated into Spanish. Leading Spanish-speaking researchers were identified through an additional search. An online survey was designed in RedCap and administered to the experts identified. The Delphi methodology was applied to obtain a consensus on the translated terms and definitions. A consensus of at least $75 \%$ was considered to be necessary to define the final ABC Taxonomy translated into Spanish.

Results: The literature search identified the following synonyms in Spanish of the ABC Taxonomy: 11 (adherence to medication), 2 (initiation), 2 (implementation), 9 (discontinuation), 10 (persistence), 3 (management of adherence) and 3 (adherence-related sciences). By the time this abstract was submitted, 54 experts had answered the survey (response rate 28\%), 69\% of them from Spain. The second Delphi round will provide the final list of Spanish synonyms and definitions.

Conclusion: The translation of the ABC Taxonomy for medication adherence into Spanish will facilitate valid comparisons between study findings.

\section{Medication management and adherence of patients with chronic diseases during the COVID-19 pandemic}

\author{
Judit Simon ${ }^{1}$, Bence Kovács ${ }^{1}$ \\ ${ }^{1}$ Corvinus University of Budapest, Institute of Marketing, Hungary \\ (judit.simon@uni-corvinus.hu)
}

Introduction: During the COVID-19 pandemic the patients with chronic diseases were more threatened by the virus, but they were more limited in access to contact the doctors.

The aim of the research: was to find out, how the patients with chronic illnesses perceived the threat of pandemic, how they changed the way of contacting the doctors and how was the medical adherence influenced by the pandemic.

Methods: A survey was conducted among 583 respondents in a panel of patients with chronic diseases with online self-administered questionnaire, built up based on the research questions. The 
perception of threat was measured by the fear of COVID-19 scale (FCV-19S).

Results and discussion: The published scale has been proven in this population as valid (Cronbach alpha 0.86 ). The patients perceived the threat of the pandemic in general very intensively, but there are some differences according to demography and health condition. About $70 \%$ of the respondents had to change the way of contacting the doctors (mainly by phone and using more e-health equipments). About $10 \%$ of the respondents changed the regimens of medicines, but the main segment tried to be more adherent as usual. We found a statistical significant relationship between medical adherence (MARS-5) and the change of medical behaviour.

Conclusion: The patients with chronic diseases perceived pandemic as a big threat for them, they improved the behaviour and only $10 \%$ changed the behaviour into a less adherent way.

\section{Introduction of a smart pillbox in Estonia: the initial feedback from healthcare specialists}

\author{
Keidong, H. ${ }^{1}$, Volmer, D. ${ }^{1}$ \\ ${ }^{1}$ University of Tartu, Institute of Pharmacy (keidong.hanna@ \\ gmail.com)
}

Introduction: To improve medication adherence of geriatric polypharmacy patients, a digital medication dispenser is being developed in Estonia. For healthcare specialists, it gives an opportunity to assess medication adherence and prevent potential drug related problems during treatment process.

Aim: The aim of this study is to evaluate perspectives of healthcare specialists on medication adherence and perception towards smart pillbox as a supportive device to monitor regular use of medications by patients. Methods: Electronic cross-sectional survey among healthcare specialists (general practitioners, nurses and pharmacists) in Estonia $(\mathrm{N}=200)$, identifying potential problems with medication adherence and expectations towards the smart device in identifying potential medication adherence problems.

Results: Initial results revealed that about $50 \%$ of geriatric patients use more than three medications daily and $90 \%$ of them have problems with medication adherence. On a scale of 1-5 (never-very often), the frequency of existing adverse drug reactions is 3 , mixing different medications is 3 , problems with finding medications is 3 and difficulty of remembering medication doses is 4 .

Discussion: Low medication adherence is seen as a problem by healthcare specialists. In developing of smart devices, it is important to monitor not only the patient's needs, but also the expectations of healthcare professionals towards measuring health indicators and transmitting information.

Conclusion: Smart systems have a great potential to improve efficiency of drug treatment and give healthcare specialists a better understanding about the extent of patient's medication adherence.

\section{Components of a structured oral anti-cancer medication program: a scoping review of the literature}

Kapeena Sivakumaran, MPH ${ }^{1}$; Pamela K. Ginex, EdD, RN, $\mathrm{OCN}^{2}$; Haya Waseem, MPH ${ }^{1}$; Sarah Lagler-Clark, MPH(c) ${ }^{1}$; Tejanth Pasumarthi ${ }^{3}$; Nicole Palmer, MPH ${ }^{4}$; Kris LeFebvre, MSN, RN, AOCN ${ }^{2}$; Rebecca L. Morgan, PhD, MPH

${ }^{1}$ Department of Health Research Methods, Evidence and Impact, McMaster University, Hamilton, Ontario, ${ }^{2}$ Oncology Nursing
Society, ${ }^{3}$ School of Interdisciplinary Science, McMaster University, Hamilton, Ontario, ${ }^{4}$ Department of Population and Quantitative Health Sciences, Case Western Reserve University School of Medicine, Cleveland, Ohio (pginex@ons.org)

Aim: Medication adherence is a multifactorial process, and a multidisciplinary approach is vital in improving patient adherence. The aim of this scoping review is to identify the components of structured oral anti-cancer medication programs and assess their impact on patient adherence.

Methods: A medical librarian searched Embase, PubMed and CINAHL from January 2000 to May 2021 for published articles on institution or practice-level oral anti-cancer medication programs. Titles and abstracts, and full-text studies were screened independently and in duplicate by two reviewers. From eligible full-text studies, study and program characteristics and components of the programs will be extracted. Results will be synthesized and reported under key conceptual categories and thematic synthesis will be used to contextualize findings where appropriate. We will identify and summarize research gaps and opportunities.

Results: Our search identified 3518 records, of which 26 studies were included in the review, with 15 distinct programs reported. Many adherence programs were multicomponent, involving patient counseling, educational sessions and home-based care. Programs were also multidisciplinary, involving oncologists, oncology nurses and pharmacists, with many being pharmacy-led.

Discussion: As cancer treatment shifts to include oral oncolytic therapies, programs to support patients on treatment and the staff caring for them are needed. Interprofessional teams can ensure patient understanding, monitor, and improve adherence, and manage side effects, which can lead to improved patient outcomes.

Conclusion: The findings from this scoping review will inform medication adherence programs and clinical practice guidelines for the oncology community.

\section{How to implement chemical adherence testing in routine clinical practice}

\section{Kably B. ${ }^{1}$, Derobertmasure A. ${ }^{1}$, Billaud E. ${ }^{1}$, Lorthioir A. ${ }^{2}$,} Amar L. ${ }^{2}$, Boutouyrie P. ${ }^{1}$, Azizi M. ${ }^{2}$

${ }^{1}$ Hopital Europeen Georges Pompidou - Pharmacology Unit, Paris, France; ${ }^{2}$ Hopital Europeen Georges Pompidou - Hypertension Unit, Paris, France (benjamin.kably@aphp.fr)

The aim of this study was to compare two methodologies for the implementation of chemical adherence testing (CAT) in routine clinical practice: "free prescription" (FP) vs. "systematic sampling" (SS).

This study was approved by an Ethical Committee.

For the FP study, we made CAT available to all prescribers, with no particular instructions.

For the SS study, all patients addressed for a first consultation to our center were screened.

The FP study lasted 9 months and included 119 patients, 53.8\% male, mean age of 54.9 years, and blood pressure (BP) of $168 / 96 \mathrm{mmHg}$.

With a median of 4.0 antihypertensive drugs, non-detection rate was $51.3 \%$ ( $16.8 \%$ of complete non-detection).

The SS study lasted 4 months and included 191 patients, $51.3 \%$ male, mean age of 57.0 years and $\mathrm{BP}$ of $138 / 92 \mathrm{mmHg}$.

With a median of 2.0 antihypertensive drugs, non-detection rate was $15.2 \%$ ( $7.0 \%$ of complete non-detection). 
Clinicians' ability to predict non-adherence was previously reported to be accurate $50 \%$ of the time, which is close to our results with the FP methodology. The SS methodology is more time- and resource-consuming but the absolute number of nonadherent patients detected was greater, in a shorter period of time.

Both methodologies were successful in the discovery of high numbers of non-adherent patients.

Human resources and lab capacities are crucial factors for the implementation of CAT in routine practice. Further studies (including medico-economic) and other possible ways of implementation will have to be explored.

\section{Adherence to medication and refills of patients with arterial hypertension}

\author{
Marineci C. D. ${ }^{1}$, Chirita C. ${ }^{1}$, Negres S. ${ }^{1}$ \\ ${ }^{1}$ Department of Pharmacology and Clinical Pharmacy, Faculty of \\ Pharmacy, University of Medicine and Pharmacy Carol Davila, \\ Bucharest, Romania (corresponding author: chirita.cornel@gmail.- \\ com) (danamarineci@gmail.com)
}

Introduction: Actual guidelines for arterial hypertension management list nonadherence to medication as one of the most important reasons for patients not attaining arterial pressure targets.

Aim: The aims of the study were to assess the level of adherence to medication of patients with hypertension and to delineate some determinants of low adherence, and possible for poor clinical outcomes.

Methods: 3095 Patients (55.8\% women) aged $62.08 \pm 13.56$ years with arterial hypertension were included. The respondents answered a questionnaire gathering sociodemographic data and other information. Adherence was assessed using the adherence to refills and medication scale (ARMS), better adherence corresponding to lower ARMS scores.

Results: Average adherence score for the entire group was $19.18 \pm 5.4$ points. Women, people living in a family and people having a higher level of education had a better adherence level compared to men, single people or people with lower education level. People taking more daily medicines and supplements had lower adherence to medication and refills (correlation coefficient $\mathrm{rs}=0.049$, $\mathrm{p}<0.05$ ). As people reported the habit of adding salt into their food more frequently, their ARMS score increased. People reporting their last measured arterial pressure being under $140 / 90 \mathrm{mmHg}$ had a smaller ARMS score $(18.34 \pm 5.19)$ compared to those having last measured arterial pressure above these values $(20.64 \pm 5.44)$.

Conclusions: Investigated Romanian hypertensive patients displayed an average level of adherence. Better adherence to medication and refills corresponded to better hypertension control.

\section{Drugs for obstructive airway diseases: prescription patterns in two different health-care settings in Piedmont (Italy)}

\footnotetext{
Armando L. G. ${ }^{1}$, Basso L. ${ }^{1}$, Traina S. ${ }^{1}$, Rolando C. ${ }^{2}$, Esiliato M. ${ }^{2}$, Cena C. ${ }^{1}$, Boscaro V. ${ }^{1}$, Miglio G. ${ }^{1,3}$

${ }^{1}$ Dipartimento di Scienza e Tecnologia del Farmaco-Università degli Studi di Torino, Italy; ${ }^{2}$ Struttura Complessa Farmacia Territoriale ASL TO4-Regione Piemonte, Italy; ${ }^{3}$ Centro di Competenza sul Calcolo Scientifico C3S-Università degli Studi di Torino, Italy (lucrezia.armando@edu.unito.it)
}

Aim: To assess whether utilization of drugs for obstructive airway diseases (ATC R03) and corticosteroids for systemic use (ATC H02) is associated with the health-care settings at the local health unit ASLTO4 (Piedmont, Italy).

Methods: To identify discrepancies in patterns of drug utilization, a comparative case-control study was conducted. Drugs prescribed over a 13-month period (June 2018-July 2019) by 10 general practitioners to 416 patients in a care setting (Casa della Salute) with a specific location, including diagnostics, medical specialists and a multidisciplinary team specific for airway diseases (cases), were compared to those prescribed to 416 matched controls found in the traditional outpatients setting of the ASLTO4 in the same period of time (controls). These data were used to create two distinct drug prescription networks, that allowed us to better identify discrepancies in (co)prescriptions.

Results: Drugs belonging to 15 and 14 pharmacological classes were prescribed to cases and controls, respectively. Long-acting beta-agonists + inhaled corticosteroids (ICS) and long-acting muscarinic antagonists, were prescribed to a significant larger subgroup of cases rather than controls. Oppositely, in comparison to cases, short-acting beta-agonists + short-acting muscarinic antagonists, ICS and xanthines were prescribed to a significant larger subgroup of controls.

Discussion: Prescription of drugs for obstructive airway diseases was associated to the health-care setting.

Conclusion: Comparison of prescribing patterns should be further investigated in the area of drugs for obstructive airway diseases.

\section{Measurement during implementation phase by integrating real-time adherence data: dose-weighted composite medication possession ratio (cMPR)}

\section{Bevan A. ${ }^{1}$, Hoo Z. H. ${ }^{1}$ \\ ${ }^{1}$ United Kingdom (amanda.bevan@uhs.nhs.uk)}

Introduction: The CFHealthHub Digital Learning Health System ( https://www.cfhealthhub.com/) is a collaboration between $15 / 24$ of the English adult cystic fibrosis (CF) centres using real-time data to improve adherence and self-care. One of its aims is to reduce medicines waste by matching inhaled medicine supplies (MPR) with actual use (objective adherence). For those on $\geq 2$ medications, cMPR is typically calculated as a mean of all individual MPR whereas objective adherence will be weighted according to medicine doses prescribed. This difference in calculation method may cause spurious discrepancy between cMPR and objective adherence.

Aim: To compare "standard" cMPR with dose-weighted cMPR in 230 adults with $\mathrm{CF}$ on $\geq 2$ inhaled medicines with $\geq 1$ year of continuous objective adherence data.

Methods: Dose-weighted cMPR was calculated by.

1 Calculating total doses by adding up the doses of all individual medicine (MPR $\times 365$ days $\times$ daily dose).

2 Calculating the weighting for each medicine as the proportion of doses per medicine from the total doses.

3 Multiplying each individual MPR by the weight calculated in step 2.

4 Adding up all values from step 3 .

Dose-weight cMPR was compared against "standard" cMPR using non-parametric methods.

Results: Among 230 adults, only 137 (59.6\%) have dose-weighted cMPR that was within $5 \%$ of "standard" cMPR. Dose-weighted cMPR exceeded "standard" cMPR by $4 \%$ (95\% CI 3-6\%) without a clear direction in bias.

Discussion: Dose-weighted and "standard" cMPR are substantially different and not inter-changeable. 


\section{Development of a new hospital medication adherence scale in cardiovascular disorders: the Mascard}

\author{
Karin Martin-Latry ${ }^{1,2,3}$, Philippe Latry ${ }^{4}$, Yann Pucheu ${ }^{1}$, \\ Thierry Couffinhal ${ }^{1,2,3}$
}

${ }^{1} \mathrm{CHU}$ de Bordeaux, Centre d'exploration, de prévention et de traitement de l'athérosclérose, 33,600 Pessac, France (karin.martinlatry@u-bordeaux.fr)

Introduction: Non-adherence to prescribed cardiovascular drugs may leads to complications in both secondary and primary prevention. Thus, cardiovascular diseases lead to regular monitoring, which may require hospitalizations, making the hospital a place to re-evaluate both the patient's health and the medication adherence behavior in primary care.

Main: The objective of this study was to develop a new scale to assess medication adherence in patients with cardiovascular diseases usable during hospitalization.

Methods: A cohort of 219 high risk cardiovascular patients was included for this study. Data on reasons for non-adherence were collected using the newly developed medication adherence scale in cardiovascular disorders (Mascard) and compared with physician assessment and the control of their cardiovascular risk factors.

Results: The Mascard consists of 5 items concerning attitudes toward medication intake. It has good psychometric properties (Cronbach alpha 0.49 ) and validity [positive predictive value (PPV) 0.96 and negative predictive value (NVP) 0.5 ] and correlated with physician assessment (Somers D $=0.34, \mathrm{P}<10-4$ ) and control of cardiovascular risk factors.

Discussion and conclusions: This new free to use and validated scale is rapid, easy to use and may be useful for health care practitioners to assess cardiovascular medication adherence in inpatients.

\section{Digital communication to assess medication experiences: needs and wishes of patients and pharmacists}

Diana Maric ${ }^{1}$, Liset van Dijk ${ }^{1,2}$, Nathalie van Schayk ${ }^{3}$, Claudia Rijcken $^{3}$, Marcia Vervloet ${ }^{2}$

${ }^{1}$ Department of PharmacoTherapy, -Epidemiology \& -Economics (PTEE), Groningen Research Institute of Pharmacy, Faculty of Mathematics and Natural Sciences, University of Groningen, The Netherlands; ${ }^{2}$ Nivel, Netherlands Institute for Health Services Research, The Netherlands; ${ }^{3}$ Pharmi B.V., The Netherlands (M.Vervloet@nivel.nl)

Aim: Early detection of problems patients experience in their medication use can improve medication adherence. The TRIAGE question set aims at detecting problems at the counter. TRIAGE could potentially be valuable to digitally guide patients between pharmacy visits, e.g. by integrating the questions into a digital pharmacist app. Our aim was to assess needs and wishes of patients and pharmacists concerning digital communication.

Methods: Semi-structured interviews were held with eight pharmacists. Two digital focus groups with 12 patients were organized. All audiotapes were transcribed. Data were analysed with open, axial and selective coding using Atlas.ti.

Results: Pharmacists and patients were positive about digital communication. Both valued the possibility of monitoring medication use by assessing experiences in between pharmacy visits through an app and considered the TRIAGE-questions to be suitable. Patients and pharmacists suggested some rephrasing of questions as well as adding a question on how the medication was used. Patients expect feedback preferably in the next pharmacy visit. Pharmacists expect that the responses entered in the app will facilitate the conversation in the pharmacy, especially for patients with negative experiences, and expect this to improve adherence.

Discussion: Selection bias might have occurred. The viewpoint of patients with limited (digital) literacy skills remains unknown.

Conclusion: Patients and pharmacists are positive about digital communication between pharmacy visits and view integration of TRIAGE-questions in a digital app, with some adjustments, as an added value for supporting patients' medication use.

Medication adherence among young adults in the nonEU country-implications from a web-based study

\section{Kamberi Fatjona $^{1}$, Enkeleda Sinaj ${ }^{2}$, Vjollca Ndreu ${ }^{3}$ \\ ${ }^{1}$ Research Centre of Public Health, Faculty of Health, University of Vlore "Ismail Qemali", Vlore Albania; ${ }^{2,3}$ Faculty of Technical Medical Sciences, University of Medicine Tirana, Albania (fatjonakamberi@gmail.com)}

Introduction: Medication adherence is a common problem among elderly adults and the literature on nonadherence is considerable for this age group but there is a gap of publications in young adults.

Aim: The aim was to assess the level of medication adherence among young adults in Albania.

Methods: A cross-sectional web-based study was conducted in May 2021. The Rief adherence index (RAI) questionnaire was used for the data collection through the Google forms platform using the snowballing method. The call for participation was posted in the authors' social networks with different reminders. Only adult people age $\geq 18$ were eligible to participate. A checklist for Reporting Results of Internet E-Surveys (CHERRIES) was taken into account while conducting the study. The participants had the possibility to complete the questionnaire once.

Results: The study included a total of 440 people. The mean age was $29.8 \pm$ SD 14.8. $77.3 \%$ of participants were female with higher education. $23.6 \%$ of participants suffered from a chronic illness. Regularity was reported in initiating the use of medications prescribed. Changing medication doses and discontinuing treatment earlier without consulting a doctor was the most common phenomenon found.

Discussion: In this study, we found that medication non-adherence is common among young adults although with high educational levels. Conclusion: Health professionals should provide sufficient information and counseling on medication adherence to doses and taking medications as prescribed to young adults that often are underestimated in relation to medication adherence.

\section{A pharmaceutical care intervention increased adherence seemingly through an effect on beliefs about medicines}

\author{
Östbring M. J. ${ }^{1}$, Petersson G. ${ }^{1}$, Eriksson T. ${ }^{2}$, Hellström L. ${ }^{1}$ \\ ${ }^{1}$ Linnaeus University, Sweden; ${ }^{2}$ Malmö University, Sweden; \\ (malin.johansson.ostbring@1nu.se) \\ Introduction: Beliefs about medicines is one of the strongest deter- \\ minants of adherence. It's plausible that adherence interventions can \\ be effective by changing patients' beliefs about medicines.
}


Aim: To describe how a pharmaceutical care intervention affected beliefs about medicines and adherence.

Methods: In the Motivational Interviewing and Medication review in Coronary heart disease (MIMeRiC) trial, 316 patients were randomized to a pharmaceutical care intervention during around 6 months, or standard care. Key secondary outcome was a combination of persistence (at least one refill in the period 12-16 months) and a self-report instrument at 15 months. Beliefs about medicines specific (BMQ-S) was used as a process measure.

Results: At follow up, $88 \%$ and $77 \%(\mathrm{P}=0.033)$ of patients were adherent in the intervention and control group respectively. Patients who had received the intervention had a more positive necessityconcern differential (NCD) than patients with standard care, 7.9 (5.7) vs. 6.3 (5.8); $\mathrm{P}=0.022$. Patients in the intervention group who were adherent had a mean NCD of 8.8 (5.6), while intervention patients who were not adherent had mean NCD 5.4 (5.5); $\mathrm{P}=0.046$. In patients receiving standard care, the mean NCD was 6.7 (5.5) and 6.3 (5.9); $\mathrm{P}=0.746$ among patients who were adherent and not adherent at follow-up.

Conclusion: The intervention seemed to increase adherence through an effect on patients' medication beliefs.

\section{Baseline drug profiles and COVID-19 mortality in Spain and Italy}

Bliek-Bueno K., Gimeno-Miguel A., Mucherino S., Aza-PascualSalcedo M., Orlando V., Carmona-Pírez J., Guarino I., Menditto E.

${ }^{1}$ EpiChron Research Group, IACS, IIS Aragón, Spain; ${ }^{2}$ CIRFF, Center of Drug Utilization and Pharmacoeconomics, University of Naples Federico II, Naples, Italy (kbliek@salud.aragon.es)

Aim: To compare baseline drug-use profiles and COVID-19 mortality in patients from the PRECOVID cohort in Aragón, Spain, and the Campania Region Database (CaReDB) in Campania, Italy.

Methods: Retrospective cohort study including all individuals with laboratory-confirmed COVID-19 infection between March 4 and April 17, 2020 in both regions, followed until May 17 or to the date of death. We analysed sociodemographic characteristics (sex, age, region), all the drugs dispensed in community and hospital pharmacies with a prevalence above $1 \%$ in the 3 months prior to inclusion in the study, and all-cause mortality. We performed age-adjusted logistic regression models to analyse the association between the presence of each drug studied at baseline and the likelihood of mortality during follow-up, by sex and region.

Results: 8570 Individuals, 4412 from Aragón (58.8\% women, mean age 67.7), 4158 from Campania (44.3\% women, mean age 52.1) were included. Drugs associated with an increased risk of mortality in COVID-19 patients in both regions and both sexes were antithrombotic agents, high-ceiling diuretics, antidepressants, antipsychotics, vitamin $\mathrm{B}_{12}$ and folic acid, and potassium-sparing agents.

Discussion: Certain drug profiles can influence mortality outcomes of COVID-19 patients, either as a proxy of underlying diseases or due to the direct intrinsic effects of their treatments.

Conclusion: Specific drugs related to the cardiovascular and central nervous systems were consistently associated with an increased risk of mortality in male and female COVID-19 patients in both Spain and Italy.
Medication adherence in patients treated with ACE inhibitors: a pilot study in Italian community pharmacies

\author{
Keber E. ${ }^{1}$, Comar C. ${ }^{1}$, Giua Marassi C. ${ }^{1}$ \\ ${ }^{1}$ Società Italiana di Farmacia Clinica (SIFAC), Cagliari, Italy \\ (enrico.keber@sifac.it)
}

Introduction: Available data on medication adherence and therapy awareness with reference to hypertensive patients attending Italian's community pharmacies appear to be scarce.

Aim: The present study aims to describe (a) the adherence to ACEinhibitors therapy (b) the degree of patients' knowledge of their own ACE-inhibitor therapy.

Materials and methods: The interview was conducted by 27 pharmacies spread throughout the Italian territory, on a sample of 220 patients. Inclusion criteria were the ACE inhibitor intake, age higher than 18, not first prescription cases. A two-section questionnaire consisting of 20 questions was used: medication adherence/persistence was assessed first, therapy awareness then. To ensure the representativeness of the sample, each clinical pharmacist recruited at least seven patients.

Results: Overall, $38.6 \%$ of subjects presented at least one critical issue in the medication adherence assessment questionnaire. Besides, $88.2 \%$ of the respondents showed at least one critical response on the knowledge of ACE inhibitor therapy.

Discussion: Medication adherence and therapy knowledge often represent a critical issue in patients taking ACE inhibitors. Although two out of three patients claimed to be adherent, fewer than one in three hypertensive patients are fully aware of the main aspects related to their medication.

Conclusions: This study reveals the need for pharmacist educational intervention in medication adherence and therapy awareness in patients treated with ACE-inhibitors.

\section{The development and testing of social support targeted messages to enhance medication adherence}

Sherwin, L. B., Matteson-Kome, M., Bechtold, M., Reeder, B. P., and Keefer, L. A.

${ }^{1}$ Sinclair School of Nursing, University of Missouri, Columbia, MO, USA; ${ }^{2}$ MU Healthcare, Columbia, MO, USA; ${ }^{3}$ Icahn School of Medicine, Mount Sinai, NY, NY, USA (sherwinl@missouri.edu)

Poor medication adherence is a public health problem associated with suboptimal clinical outcomes. A variety of social support methods enhance medication adherence; however, social support delivery from the patients' personal support network through virtual text messaging has not been well studied. Moreover, the mechanisms by which social support increases adherence have not been identified. Aim: This ongoing study is designed to develop text message exemplars perceived as supportive of medication adherence, and evaluate the messages for content validity, perceived levels of social support, and motivation to enhance medication adherence.

Methods: Using a mixed-methods study design, participants and their social support designate will be interviewed separately to obtain exemplars of comments and actions perceived as supportive and motivational toward enhancing medication adherence. Interviews will be audiotaped, transcribed verbatim, and analyzed using inductive content analysis. A portfolio of supportive medication adherence messages will be generated. Medication adherence experts will evaluate the portfolio for content validity and recommend revisions as 
needed. Participants will quantitatively rate the usefulness, perceived level of social support, and perceived level of motivation of each message from the portfolio using a standard 0 to 10 numeric rating scale.

Results: Funding and Institutional Review Board approval have been obtained. Participant recruitment is underway.

Discussion/conclusion: The resulting portfolio will become components of a tailored virtual text messaging social support mHealth intervention to enhance medication adherence in an extramurally funded study.

\section{On TRACk: through training, preparation, and counseling, to better use of inhaled medication}

\author{
te Paske R. ${ }^{1}$, Linn A. J. ${ }^{1}$, van Boven, J. F. M. ${ }^{1}$, van Dijk, L. ${ }^{1}$, \\ Vervloet, M. $^{1}$ \\ ${ }^{1}$ Netherlands (r.tepaske@nivel.nl)
}

\begin{abstract}
Aim: Over $70 \%$ of patients with asthma/COPD in the Netherlands make mistakes in inhaling their medication. The community pharmacy plays a vital role in medication education. We aimed to develop and pilot the On TRACk-intervention: an online communication training for pharmacy team members (PTs) on inhaled medication counseling and counseling preparation for patients and PTs to improve medication adherence and health outcomes in patients with asthma/COPD.

Methods: The On TRACk internet portal was developed to facilitate PT's communication training and PTs' and patients' counseling preparation. The intervention was piloted in two community pharmacies, with 4 PTs and 13 asthma/COPD patients. Interviews were conducted to evaluate their experiences.

Results: PTs acknowledged that the personal feedback they received in the communication training was applicable in daily practice. They valued suggestions to stimulate patient involvement, and to provide tailored information that matches their patients' needs. Patients valued the opportunity to propose topics they considered significant to discuss and felt more engaged.

Discussion: The pilot was successful. On TRACk improves PTs' inhaler medication counseling skills and bolsters patients' involvement in their medication use. However, On TRACk needs to be evaluated in the upcoming clinical trial on its effectiveness on medication adherence and patients' health outcomes.

Conclusion: The On TRACk intervention aligns with PTs' needs and wishes. Counseling preparation provides patients with the opportunity to explore their own barriers in medication use and empower them in consultations with healthcare providers.
\end{abstract}

\section{Dispensing data are inconclusive to detect inadequate real-life implementation of oral anticoagulants: a proof- of-concept analysis}

\author{
Dietrich F. ${ }^{1}$, Lehner A. ${ }^{1}$, Barbati S. ${ }^{1}$, Hersberger K. E. ${ }^{1}$, Arnet I. ${ }^{1}$ \\ ${ }^{1}$ Pharmaceutical Care Research Group, Department of Pharmaceuti- \\ cal Sciences, University of Basel, Switzerland \\ (fine.dietrich@unibas.ch)
}

Introduction: For stroke patients with atrial fibrillation, direct oral anticoagulants (DOAC) are taken for life to prevent recurrent events. New approaches are required to identify non-adherent behaviors in real-life settings.
Aim: To investigate whether inadequate adherence to DOAC measured by electronic monitoring is reflected in dispensing data. Methods: We used patient data of the ongoing MAAESTRO study and compared six months of electronic monitoring with corresponding dispensing data. Dispensing data were collected from community pharmacies and self-dispensing physicians. We assessed "taking adherence," "timing adherence," and "drug holidays" (three consecutive days without intake). We defined dispensing patterns according to early refills or gaps following late refills.

Results: Electronic data from twelve patients (mean age $77.8 \pm 7.2$ years, $41.7 \%$ female) showed a median "taking adherence" of $89.7 \%$, IQR $74.7-95.8$ (above $90 \%$ for five patients). Ten patients presented an adequate refill behavior. Electronic monitoring revealed the inadequate behavior "drug holidays" for four patients, from whom three showed the pattern "refills too early" and one was congruent to "gaps in the middle of refills".

Discussion: The concept to use dispensing patterns to detect inadequate implementation of DOAC seems feasible, but the results are inconclusive. Our analysis is ongoing with a larger population.

Conclusion: Dispensing patterns are easy to define but lack the ability to unveil nuanced shapes of non-adherence compared to electronic monitoring.

\section{The ENABLE Online Repository of medication adherence technologies: early phase results}

\author{
Nabergoj Makovec U. ${ }^{1}$, Goetzinger C. $^{2}$, Ribaut J. $^{3}$, \\ Haupenthal F. ${ }^{4}$, Herdeiro M. T. ${ }^{5}$, Jácome C. ${ }^{6}$, Roque F. ${ }^{7}$, \\ Smits D. ${ }^{8}$, Tadic I. ${ }^{9}$, Dima A. L. ${ }^{10}$
}

${ }^{1}$ University of Ljubljana, Faculty of Pharmacy, Department of Social Pharmacy, Ljubljana, Slovenia; ${ }^{2}$ Department of Population Health, Luxembourg Institute of Health, Strassen University of Luxembourg, Faculty of Science, Technology and Medicine, Luxembourg; ${ }^{3}$ Institute of Nursing Science, Department Public Health, Faculty of Medicine, University of Basel \& Department of Theragnostic, Hematology, University Hospital of Basel, Switzerland; ${ }^{4}$ Division of Nephrology and Dialysis, Department of Medicine II, Medical University of Vienna, Austria; ${ }^{5}$ Institute of Biomedicine, Medical Sciences Department, University of Aveiro, Aveiro, Portugal; ${ }^{6}$ Department of Community Medicine, Information and Health Decision Sciences (MEDCIDS) \& Portugal Center for Health Technology and Services Research (CINTESIS), Faculty of Medicine, University of Porto, Porto, Portugal; ${ }^{7}$ Research Unit for Inland Development, Polytechnic of Guarda, Guarda, Portugal; ${ }^{8}$ Faculty of Public Health and Social Welfare, Department of Public Health and Epidemiology, Riga Stradins University; Riga, Latvia; ${ }^{9}$ University of Belgrade, Faculty of Pharmacy, Department of Social Pharmacy and Pharmaceutical Legislation, Belgrade, Serbia; ${ }^{10}$ Université Claude Bernard Lyon 1, Research on Healthcare Performance (RESHAPE), INSERM U1290, Lyon, France (urska.nabergoj.makovec@ffa.unilj.si)

Introduction and aim: Numerous technologies exist to support medication adherence, yet few are implemented into practice. An online interactive repository of available technologies may facilitate their selection and adoption by different stakeholders. Developing such repository is among the main objectives of the ENABLE COST Action (CA19132). However, meeting the needs of diverse stakeholders requires careful considerations of descriptive entities.

Methods: Eleven members with research, practice, and policy background from nine European countries formed an Interdisciplinary Steering Committee. The ontology development recommendations by Wright et al. (2020) were followed to define the scope, descriptive 
entities, their labels and definitions. Hence, a list of descriptors was developed iteratively through literature review, discussions within the Steering Committee and with other ENABLE Action members. Following, the descriptors will be evaluated by relevant stakeholders in a real-time online Delphi survey.

Results: Behaviour, implementation, and computer sciences inform the repository development to ensure interoperability with existing ontologies and information sources. Fifteen descriptor groups are proposed: adherence management goals; target user, condition, medication, regimen and product characteristics; mode of delivery; measurement type; intervention target determinants, behaviour change techniques, provider and setting; health policy; implementation strategies and quality indicators.

Discussion: Interoperability with existing definitions, ontologies, and e-Health frameworks as well as considerations of different stakeholder needs are the main elements reflected in the proposed descriptors.

Conclusion: The proposed descriptors form a foundation for further stakeholder consultation on the pathway to create an evidence based and user-friendly repository.

\section{What is in the shadows matters! Antecedents and self- efficacy on adherence}

Maffoni M. MPsych PhD ${ }^{1}$, Pierobon A. MPsych ${ }^{1}$, Granata N. MPsych ${ }^{2}$, Vigorè M. MPsych ${ }^{1}$, Giardini A. MPsych ${ }^{3}$

${ }^{1}$ Istituti Clinici Scientifici Maugeri, IRCCS, Psychology Unit of Montescano Institute, Italy; ${ }^{2}$ Istituti Clinici Scientifici Maugeri, IRCCS, Department of Cardiac Respiratory Rehabilitation of Tradate Institute, Varese, Italy; ${ }^{3}$ Istituti Clinici Scientifici Maugeri, IRCCS, IT Department, Pavia Italy (marina.maffoni@icsmaugeri.it)

Aim: This work presents a self-reported schedule which investigates both patients' self-efficacy and adherence antecedents.

Methods: Based on literature review and clinical experience, an Italian interdisciplinary group developed tools to investigate patients' medication adherence (ASiA: Adherence Schedule in Asthma; ASiCOLD: Adherence Schedule in Chronic Obstructive Lung Disease; ASiHF: Adherence Schedule in Heart Failure; ASiHD: Adherence Schedule in Ischemic Heart Disease; ASiT: Adherence Schedule in Transplantation). These schedules were collectively discussed in order to define a non-disease specific schedule on patient's self-efficacy, and cognitive emotional antecedents of adherence.

Results: The resulting instrument "ASonA" (Antecedents and SelfEfficacy on Adherence Schedule), composed by 21 items, comprises 3 subscales: Antecedents (ASonA-A) - health literacy, disease understanding, social support perception and health-related limitations acceptance; Self-efficacy (ASonA-SE) - beliefs and self-care strategies; Affectivity (ASonA-Aff) - emotional response related to the health condition. The ASonA was satisfactorily adopted among Italian and Polish chronic patients with hypertension (BEL4ADHER, https://doi.org/10.2147/PPA.S258999) and it is currently employed in an Italian financed prospective observational study on cognitive impairment, frailty and rehabilitation outcome in patients with cardiorespiratory disease (DEC_FRAinRIAB; Grant Number 2424_20 04 20). Preliminary data will be presented.

Discussion: This instrument shows a good usability in research and clinical settings, focusing on patients' features potentially affecting all phases of the adherence process.

Conclusion: This rapid, self-reported and transdiagnostic instrument can unveil specific risk factors for adherence, inspiring tailored psychoeducational interventions to support patients to adhere along all phases of the medication treatment.
Electronically monitored medication adherence and behavioural patterns of direct oral anticoagulants

Kolmanova E. ${ }^{1}$, Dvorackova S. ${ }^{1}$, Sisakova M. ${ }^{2}$, Brabec M. ${ }^{2}$, Penka M. ${ }^{3}$, Kala P. ${ }^{2}$, Maly J. ${ }^{\text {, }}$, Mala-Ladova K. ${ }^{1}$

${ }^{1}$ Department of Social and Clinical Pharmacy, Faculty of Pharmacy in Hradec Kralove, Charles University, Czech Republic; ${ }^{2}$ Department of Internal Medicine and Cardiology, University Hospital Brno and Medical Faculty of Masaryk University, Brno, Czech Republic; ${ }^{3}$ Department of Clinical Hematology, University Hospital Brno and Medical Faculty of Masaryk University, Brno, Czech Republic (kolmanoe@faf.cuni.cz)

Aim: Several aspects must be considered to consolidate our understanding of patients' views and attitudes to prescribed medicines while capturing the medication adherence (MA). The aim of this study was to analyse MA to long-term use of direct oral anticoagulants (DOACs) in patients with atrial fibrillation focusing mainly on the medication taking and timing regimen.

Methods: This is a prospective single-centre follow-up study conducted in the University Hospital in the Czech Republic. It comprises adult outpatients with atrial fibrillation, who are treated with DOACs at least 3 months. Patients are invited for an initial structured interview and two follow-up visits after 3 and 6 months. Self-reported MA and beliefs about medicines are measured by Czech validated questionnaire tools (MARS-CZ, BMQ-CZ) as well as awareness patterns about taking DOACs are evaluated at the first visit. The Medication Event Monitoring System buttons attached to medication dispenser or pillbox are employed to electronically monitored implementation and persistence phases of MA during the whole observing period.

Results: This is an ongoing study and result analysis is in progress. Electronically monitored MA and behavioural patterns including selfreported MA from the initial study visit will be presented in expected sample of 100 patients.

Discussion and conclusion: This is the first complex study conducted in the Czech Republic in patients with DOACs and it is expected to obtain a unique set of data about the medication taking behaviour including electronic buttons technology.

\section{Medication adherence in patients with hemophilia on prophylaxis born before and after 1960}

Bago M. ${ }^{1}$, Butkovic A. ${ }^{2}$, Preloznik Zupan I. $^{3}$, Faganel Kotnik B. ${ }^{3}$, Prga I. ${ }^{1}$, Bacic Vrca V. ${ }^{4}$, Zupancic Salek S.

${ }^{1}$ Andrija Stampar Teaching Institute of Public Health Zagreb, Croatia; ${ }^{2}$ University of Zagreb, Croatia; ${ }^{3}$ University Medical Center Ljubljana, Slovenia; ${ }^{4}$ Clinical Hospital Dubrava Zagreb, Croatia; ${ }^{5}$ University Hospital Centre Zagreb, Croatia (martina.bago.hr@gmail.com)

Introduction: Advances in treatment of hemophilia after 1960 have led to the increase of life expectancy among patients with hemophilia. There is limited research into specific characteristics of ageing patients with hemophilia. Aim of this study was to examine characteristics of patients with hemophilia on prophylaxis from Croatia and Slovenia born before 1960 and compare them to the ones born after 1961.

Methods: Implementation phase of medication adherence was assessed with self-reported VERITAS-Pro instrument, depressive symptoms with BDI-II and health related quality of life with SF-36v2. Results: A total of 82 participants were included in the study, 13 born before 1960, 69 born after 1961. Only 1 patient (7.7\%) born before 1960 had a score above cut-off point for non-adherence, while 13 
patients $(18.8 \%)$ born after 1961 had a score indicating non-adherence. Significant differences were found between two groups for the total adherence score and remember subscale, depressive symptoms and physical component summary.

Discussion: Ageing patients with hemophilia in Croatia and Slovenia exhibit better medication adherence scores, but more depressive symptoms and lower physical health related quality of life.

Conclusion: Results indicate that medication adherence is good among older patients with hemophilia. However, problems with depression and health related quality of life, which are probably both age and disease related, have to be taken into consideration.

\section{Medication management for non-communicable diseases during the COVID-19 outbreak: a snapshot on the European situation}

Kardas P. ${ }^{1}$, van Boven J. F. M. ${ }^{2}$, Wettermark B. ${ }^{3,4}$, Menditto E. ${ }^{5}$, Pinnock H. ${ }^{6}$, Tsiligianni I. ${ }^{7}$, Agh T. ${ }^{8}$

${ }^{1}$ Medication Adherence Research Centre, Department of Family Medicine, Medical University of Lodz, Poland; ${ }^{2}$ Department of Clinical Pharmacy \& Pharmacology, Medication Adherence Expertise Center of the Northern Netherlands (MAECON), University Medical Center Groningen, University of Groningen, The Netherlands; ${ }^{3}$ Department of Pharmacy, Faculty of Pharmacy, Uppsala University, Sweden and ${ }^{4}$ Faculty of Medicine, Vilnius University, Lithuania; ${ }^{5}$ Department of Pharmacy, CIRFF, Center of Pharmacoeconomics and Drug Utilization Research, University of Naples Federico II, Italy; ${ }^{6}$ Usher Institute, University of Edinburgh, United Kingdom; ${ }^{7}$ Department of Social Medicine, School of Medicine, University of Crete, Crete, Greece; ${ }^{8}$ Syreon Research Institute and Division of Pharmacoeconomics, Department of Pharmaceutics, Faculty of Pharmacy, University of Pecs, Hungary (tamas.agh@syreon.eu)

Aim: To evaluate medication management for non-communicable diseases (NCDs) during the second wave of the COVID-19 pandemic across Europe and association with COVID-19 burden and country income.

Methods: In December 2020, COST Action ENABLE (CA19132) conducted a cross-sectional, web-based survey in 38 European countries and Israel on available face-to-face and e-health medication management services in place for NCDs. COVID-19 burden was defined as the number of positive cases and deaths per 100,000 inhabitants; country income was assessed as gross domestic product per capita (GDP/capita) at purchasing power parity. Descriptive statistics, nonparametric tests and generalized linear models were applied in this analysis.

Results: 53 Experts from 39 countries completed the survey. In 35 $(90 \%)$ of countries face-to-face consultations were limited and this showed a positive association with the number of COVID-19 cases $(p=0.03)$ and deaths $(p=0.05)$. The mean $(S D)$ number of available e-health services (including e-mail, online chat, phone, videoconference, electronic health record portal or other) was 3 (1.3) and this was positively associated with GDP/capita of countries $(p=0.05)$. There was no further association between the evaluated variables.

Discussion: Although COVID-19 limited the number of face-to-face appointments in NCD care, it accelerated e-health solutions.

Conclusion: Reduction in face-to-face consultations was associated with greater burden of COVID-19; however, the uptake of e-Health services was associated with higher country income. This suggest significant disparities in maintaining services for NCDs across Europe during the second wave of the pandemic.
Italian Pharmacists and COVID-19: narratives as a tool to describe the experiences during pandemic

\section{Daniela Scala $^{1}$, Stefania Polvani ${ }^{2}$, Maria Ernestina Faggiano ${ }^{3}$ \\ ${ }^{1}$ AORN “A. Cardarelli", Napoli-Coordinatore Area Scientifica SIFO "Informazione Scientifica, Educazione ed Informazione Sani- taria", Italy; ${ }^{2}$ Azienda USL Toscana Sud Est-Presidente SIMeN (Societa italiana di medicina narrativa), Italy; ${ }^{3} \mathrm{AOU}$ Policlinico di Bari-Consiglio Direttivo SIFO, Italy (sdaniela2000@yahoo.com) Introduction: During the COVID-19 pandemic, Italian pharmacists have been called on to face the emergency in a changing scenario in which their tools have become preventive measures of fatal outcomes. It was necessary to adopt alternative qualitative ways to explore the wide range of unique, personal experiences of this pandemic, and to provide a tool which allows, not only 'counting', but also 'description'.}

Aim: To collect and analyse narratives highlighting the weaknesses and strengths of Italian pharmacists in an "emergency".

Methods: Qualitative study based on the methodology of narrative medicine. Narratives were anonymously collected in the form of a free story from 16/March to 30/April 2020. Narratives were analyzed according to the classifications of Kleinman, Frank and Launer and Robinson together with transitional analysis (TA).

Results: Twenty-three narratives were collected and analysed. Findings show that in emergency situations, the NHS pharmacists work predominantly as Adult according to the State of Ego's analysis; they examine the situation, evaluate it and behave accordingly. This result, together with the data that most of the narratives are in progression and the presence of quest narratives (43.5\%), confirms that pharmacists perceive themselves as professionals (and human-beings) in evolution.

Conclusion: This study underlines the importance of educating the Italian pharmacist into Medical Humanities and the qualitative research field. The use of qualitative research is of crucial importance in order to produce "quality data", to give "meaning and color" to quantitative ones.

\section{Sustainability of digital solutions for medication adherence. A free market synopsis}

\author{
Ivan Siluan', Petrescu Andrei ${ }^{1}$, Velescu Bruno ${ }^{1}$ \\ ${ }^{1}$ Romania (ivan.siluan95@gmail.com)
}

Aim: Medication adherence is a key process for therapeutics outcomes, but many patients do not take their medication properly leading to several issues such as inefficacy or even mortality. In the last years the growth of mobile apps for medication management has significantly increase, creating a new market for patients. This study aims to assess the common features provided by mobile medication apps in order to increase medication adherence.

Methods: We carried out a review of free available medication tracking mobile apps, selected by the download metrics (over $50 \mathrm{k}$ ). The evaluation process of the facilities offered was performed in order to gain a perspective on their functionality as tools for medication adherence.

Results: The primary results pointed out the medication posology, reminder and inventory functions and user-friendly interface as main features. There were identified statistically significant difference $(p<0.05)$ in terms of downloads and users feed-back (over $4 / 5$ stars) for those apps which add to the panel of features e-health solutions (real time connection to pharmacies and clinicians). 
Discussion: Most of the apps free available at this point offers strong features in order to improve medication adherence for patients with a growth downloading trend.

Conclusion: Medication adherence apps appear to be a sustainable and free solution for early adopters, fulfilling patient needs and important perspectives in therapeutics outcomes, in every phase of medication adherence (initiate, implement and persist).

\section{EUREcA: survey on reimbursement pathways for medication adherence enhancing interventions across Europe: study protocol}

\section{Agh T. ${ }^{1}$, Hadziabdic M. O. ${ }^{2}$, Garuoliene K. ${ }^{3}$, Gregorio J. ${ }^{4}$, Aleksic V. V. ${ }^{5}$, Mevsim V. ${ }^{6}$, Jakab I., Kardas P. ${ }^{7}$}

${ }^{1}$ Syreon Research Institute and Division of Pharmacoeconomics, Department of Pharmaceutics, Faculty of Pharmacy, University of Pecs, Hungary; ${ }^{2}$ Centre for Applied Pharmacy, Faculty of Pharmacy and Biochemistry, University of Zagreb, Croatia; ${ }^{3}$ Pharmacy Center, Institute of Biomedical Science, Faculty of Medicine, Vilnius University, Lithuania; ${ }^{4}$ CBIOS-Universidade Lusófona's Research Center for Biosciences \& Health Technologies, Portugal; ${ }^{5}$ The Republic of Srpska Agency for Certification, Accreditation and Quality Improvement in Health Care, Bosnia and Herzegovina; ${ }^{6}$ Department of Family Medicine, Faculty of Medicine, Dokuz Eylul University, Turkey; ${ }^{7}$ Medication Adherence Research Centre, Department of Family Medicine, Medical University of Lodz, Poland (tamas.agh@syreon.eu)

Aim: Current literature lacks detailed understanding of the reimbursement framework of medication adherence enhancing interventions (MAEIs). As part of the ENABLE COST Action, the EUREcA (EUropean REimbursement strategies for interventions targeting medication Adherence) study aims to fill in this gap and provide an in-depth overview of reimbursed MAEIs currently available in European countries at national and regional levels.

Methods: The questionnaire for this cross-sectional, web-based survey was developed as a result of an iterative process of discussion informed by a desk review. Four external experts helped in the validation process. This survey will be reported according to the CHERRIES guideline.

Results: The desk review proved that the current literature is scarce in addressing reimbursement policies of MAEIs. Therefore, a 15-item questionnaire exploring details of reimbursed MAEIs was developed. Important concepts were explained in the introduction to ensure that respondents share the same theoretical frame. The survey will be available online in June 2021 and will target ENABLE Collaborators (mainly healthcare provider, competent authorities' professionals, and academics with a medical or pharmaceutical background) in 38 European countries and Israel.

Discussion: Providing a snapshot of the current European reimbursement landscape of MAEIs, EUREcA will help to identify availability of reimbursement models as well as gaps.

Conclusion: Our findings will create firm background for the discussion on the implementation of MAEIs and the development of related reimbursement models to be part of health or medicine policies in Europe.

\section{Systematic literature review on medication adherence of multimorbid elderly women}

\section{Erdősi D. ${ }^{1,2}$, Inotai A.,3, Vajda $\mathrm{P}^{4},{ }^{2,}$ Ágh $\mathrm{T}^{3}$}

${ }^{1}$ University of Pécs, Faculty of Pharmacy, Department of Pharmacoeconomics, Pécs, Hungary; ${ }^{2}$ Semmelweis University, Center for Health Technology Assessment; Budapest, Hungary; ${ }^{3}$ Syreon Research Institute, Budapest, Hungary; ${ }^{4}$ University of Pécs, Faculty of Pharmacy, Department of Pharmaceutics, Pécs, Hungary (erdosi.dalma@pte.hu)

Introduction: Compared with men, women generally live longer, and tend to have more diagnosed chronic conditions, which often requires lifelong, complex medication regimen. Medication non-adherence may have a major impact on clinical and economic outcomes of this multimorbid population.

Aim: To provide an overview on empirical evidence of adherence to polypharmacotherapy in multimorbid elderly women.

Methods: A systematic literature search of English-language studies published in the last 10 years was conducted in January 2021 using PubMed, Embase, Academic Search Complete and CINAHL databases. Search strategy was built up as a combination of search string on synonyms of adherence, medication, polypharmacy, multimorbidity, elderly women and postmenopause. Data on medication adherence both as explanatory and/or outcome variables were extracted.

Results: Of the 1607 records screened, 24 articles were included in the final qualitative synthesis: 13, 2 and 1 studies addressed adherence as outcome, explanatory and outcome, and explanatory variables, respectively. Eight studies reported descriptive statistics on elderly women's medication adherence. Majority of studies evaluated adherence as an outcome variable suggesting that women tend to be more adherent than men; however, aging and having more comorbidities decreased adherence. As explanatory variable, medication adherence was assessed in patients with hip fracture, depression and in a mixed-disease population study.

Discussion: Our study identified limited evidence for adherence to polypharmacotherapy in multimorbid elderly women.

Conclusion: A better understanding is needed on the influencing factors and consequences of medication non-adherence to develop effective adherence interventions for this vulnerable patient population.

\section{Implementation of a medication adherence intervention for chronic leukemia patients}

Muluneh, Benyam ${ }^{1}$; Macker, Emily ${ }^{1}$; Bryant, Ashley Leak ${ }^{1}$; Wood, William $^{1}$; Wheeler, Stephanie ${ }^{1}$; Lafata, Jennifer Elston ${ }^{1}$

${ }^{1}$ University of North Carolina, Duke University (bmuluneh@unc.edu)

Introduction/aim/methods/reintroduction: Oral anticancer agents have revolutionized clinical outcomes for chronic leukemia patients; however adherence is suboptimal.

Aim: Enhance the adoption of our adherence intervention through the development of implementation strategies for a sustainable and scalable adherence intervention for CL patients. 
Methods: We will identify barriers and facilitators to implementing an effective intervention to support medication adherence among patients with chronic leukemias (aim 1); enhance adoption of the evidence-based intervention by developing implementation strategies linked to identified barriers (aim 2); and evaluate the acceptability, appropriateness, and feasibility of the adherence intervention and implementation strategies (aim 3).

Results: The expected outcomes of this project will yield an enhanced adherence intervention with implementation strategies. Outcomes will include implementation, dissemination, and evaluation of our adherence intervention in chronic leukemia patients taking oral anticancer agents. Given the rise in the use of oral anticancer agents across many cancer types, this intervention can be adapted and tested for other malignancies including breast cancer, lung cancer, prostate cancer, renal cell carcinoma, and many more. Additionally, given known racial, ethnic, and geographic inequities in cancer-partially driven by disparities in rates of adherence to oral anticancer agentsour adherence intervention can be tested as a potential solution for achieving equitable cancer care.

Conclusion: The final adherence intervention has a high potential of translation by benefiting not only chronic leukemia patients but other cancer patients on oral anticancer.

\section{Drug use and healthcare services access in chronic patients during the COVID-19 pandemic in Italy}

\section{Manuela Casula ${ }^{1}$, Carla Carnovale ${ }^{2}$, Lorenza Scotti ${ }^{3}$, Enrica Menditto ${ }^{4}$, Elisabetta Poluzzi ${ }^{5}$}

${ }^{2}$ Unit of Clinical Pharmacology, Department of Biomedical and Clinical Sciences L. Sacco, "Luigi Sacco" University Hospital, University di Milano, Milan, Italy; ${ }^{3}$ Department of Translational Medicine, Università degli studi del Piemonte Orientale, Novara, Italy; ${ }^{4}$ Center of Drug Utilization and Pharmacoeconomics (CIRFF),
Department of Pharmacy, Federico II University of Naples, Naples, Italy; ${ }^{5}$ Department of Medical and Surgical Sciences, University of Bologna. via Irnerio 48, Bologna, Italy (manuela.casula@unimi.it)

The severe acute respiratory syndrome coronavirus 2 (SARSCoV-2) and resultant COVID-19 pandemic has brought major challenges to healthcare systems and public health policies. Reduced access to non-emergent and elective care may have resulted in increased risk of discontinuation of chronic disease management.

Our project aims at analysing this issue in several cohorts of patients with chronic disease therapies, identified from pharmaceutical administrative databases of different Italian regions (in the adult population: cardiovascular treatments, anti-depressive/anti-psychotic drugs, therapies for chronic respiratory illness, hypothyroidism or hyperthyroidism, osteoporosis, autoimmune diseases; in paediatric population: drugs for dysmetabolic syndromes, neurological and psychiatric disorders, inflammatory bowel disease).

The volume of selected drugs' prescriptions and outpatient services from January 2020 will evaluated considering time periods defined by the pandemic timing and regulatory interventions, and compared with the same periods in 2019. For each chronic disease, the variation in health care access will be assessed both at regional level, estimating the variation in drug consumption [e.g., number of defined daily doses (DDD) per 1000 inhabitants per day] and of health services use (number of prescriptions for specialist visits or examinations), and at patient level, evaluating the continuity of the treatment and the access to outpatient services necessary for the disease management.

The results of this study may drive an optimization of chronically treated population management programs, both immediately and in a future scenario after COVID-19.

Publisher's Note Springer Nature remains neutral with regard to jurisdictional claims in published maps and institutional affiliations. 